\title{
Surficial Deposits in the Bear Lake Basin
}

By Marith C. Reheis, ${ }^{1}$ Benjamin J.C. Laabs, ${ }^{2}$ Richard M. Forester, ${ }^{1}$ John P. McGeehin, ${ }^{3}$ Darrell S. Kaufman, ${ }^{2}$ and Jordon Bright ${ }^{2}$

${ }^{1}$ U.S. Geological Survey, Federal Center, Box 25046, MS-980, Denver, CO 80225

${ }^{2}$ Department of Geology, Northern Arizona University, P.O. Box 4099, Flagstaff, AZ 86011-4099

${ }^{3}$ U.S. Geological Survey, National Center, 12201 Sunrise Valley Drive, MS-981, Reston, VA 20192

\section{Open-File Report 2005-1088}

U.S. Department of the Interior

U.S. Geological Survey 
U.S. Department of the Interior

Gale A. Norton, Secretary

U.S. Geological Survey

Charles G. Groat, Director

U.S. Geological Survey, Reston, Virginia 20192

Revised and reprinted: 200x

For product and ordering information:

World Wide Web: http://www.usgs.gov/pubprod

Telephone: 1-888-ASK-USGS

For more information on the USGS-the Federal source for science about the Earth, its natural and living resources, natural hazards, and the environment:

World Wide Web: http://www.usgs.gov

Telephone: 1-888-ASK-USGS

Any use of trade, firm, or product names is for descriptive purposes only and does not imply endorsement by the U.S. Government.

Although this report is in the public domain, permission must be secured from the individual copyright owners to reproduce any copyrighted material contained within this report.

This report has not been reviewed for stratigraphic nomenclature. 


\title{
Surficial Deposits in the Bear Lake Basin
}

\author{
By Marith C. Reheis, Benjamin J.C. Laabs, Richard M. Forester, John P. McGeehin, \\ Darrell S. Kaufman, and Jordon Bright
}

\section{Introduction}

Mapping and dating of surficial deposits in the Bear Lake drainage basin were undertaken to provide a geologic context for interpretation of cores taken from deposits beneath Bear Lake, which sometimes receives water and sediment from the glaciated Bear River and sometimes only from the small drainage basin of Bear Lake itself. Analyses of core sediments by others are directed at (1) constructing a high-resolution climate record for the Bear Lake area during the late Pleistocene and Holocene, and (2) investigating the sources and weathering history of sediments in the drainage basin. Surficial deposits in the upper Bear River and Bear Lake drainage basins are different in their overall compositions, although they do overlap. In the upper Bear River drainage, Quaternary deposits derived from glaciation of the Uinta Range contain abundant detritus weathered from Precambrian quartzite, whereas unglaciated tributaries downstream mainly contribute finer sediment weathered from much younger, more friable sedimentary rocks. In contrast, carbonate rocks capped by a carapace of Tertiary sediments dominate the Bear Lake drainage basin.

Information on the type, composition, age, and distribution of surficial deposits can be used in several ways to understand changes in streamflow and lake level. First, the distribution and age of deposits such as glacial deposits, landslides, and stream terraces tell us when large amounts of sediment were eroded from hillslopes, transported by streams, and potentially deposited in Bear Lake. Second, careful mapping of marsh, lake, and river deposits between modern Bear Lake and the Bear River (fig. 1) provides information on how the lake and river have interacted over time scales of the past several thousand years to the past half-million years. Surficial mapping, radiocarbon ages, and amino-acid racemization ages (Laabs, 2001; Laabs and Kaufman, 2003; and data in this report) suggest that Bear Lake has expanded and contracted several times in the last 10,000 years and that it may have overflowed northward through channels on the west side of the valley. During this time, Bear River migrated northward through the intervening marsh to its present position. Some of these changes may be related to climate change but others probably were caused by faulting or tilting of the valley floor.

This report presents information obtained from stratigraphic sections described from outcrops and auger holes (fig. 1) in the area around Bear Lake. Stratigraphic sections measured by Reheis are graphically portrayed on Figures 2 and 3. Sections measured by Laabs and Kaufman are shown graphically in Laabs (2001). Table 1 presents locations and descriptive information for sediments that were sampled from all of the measured sections as well as from a few supplementary sites. Table 2 presents radiocarbon ages from samples of lake, marsh, and alluvial deposits. This information, combined with age data of Laabs (2001) and Laabs and Kaufman (2003), provides the stratigraphic and chronologic basis for a surficial geologic map of the Bear Lake basin (Reheis, unpub. data). Table 3 presents ostracode identifications and interpreted paleoenvironments for selected samples. The stratigraphic and paleoenvironmental data can be used in concert with much more detailed information from analyses of the Bear Lake cores to reconstruct the lake-level history and the changing relation between Bear Lake and Bear River.

In addition to the surficial mapping, dust traps were constructed at three sites around the shoreline of Bear Lake to sample the annual vertical dust deposition to the lake area. These data (table 4) provide an estimate of the modern aerosolic inputs to help interpret paleohydrologic history from the chemistry and mineral content of lake-sediment cores.

\section{Methods}

Mapping surficial deposits in such a large area $\left(2,000 \mathrm{~km}^{2}\right)$ usually relies heavily on interpretation of features visible in aerial photographs combined with field investigations. In this study, field work also was hampered by the difficulty in obtaining access to private land and by the abundant vegetation ( 100 percent cover on lower slopes and valley floor) in this relatively moist, agricultural area. Deposit types such as river terraces of different heights and channels incised into lake plains (fig. 1) were tentatively identified on aerial photographs. Locations where deposits with certain surface expressions were exposed in road or canal cuts then were visited to examine the sedimentary layers and surface features. Field work also revealed how a particular 
type of deposit is related to another in time and space; for example, lake deposits that overlie fluvial deposits indicate a rise in lake level, potentially caused by either subsidence along faults, an increase in effective moisture (runoff), or river migration toward the lake. Where no exposures were available, a bucket auger was used to obtain sediment in 10-20-cm depth increments. Auger holes usually terminated in loose sand or gravel beds that could not be cored by hand. Materials such as shells or organic matter, suitable for radiocarbon dating and for interpretation of hydrologic environments, were obtained from outcrop exposures and auger sediment. In the latter case, where sediment might be mixed by collapse within the auger hole, care was taken to ensure that shells chosen for dating were representative of the beds encountered during augering by selecting shells from within intact large (2-5 cm diameter) clods of sediment.

Shell fragments were isolated from clay by soaking in a weak Calgon solution for several hours. The solution then was poured over a 1,000- $\mu \mathrm{m}$ sieve; the shell fragments retained on the sieve were hand picked and thoroughly rinsed in distilled water. When necessary, the shells were sonicated in distilled water for 1 hour to remove additional surface sediment. In certain cases, when the shell material was still encrusted in sediment or the shells showed signs of surface alteration, the sample was soaked in dilute $(0.1 \mathrm{M}) \mathrm{HCl}$ to etch the surfaces clean.

The dust-trap design (Reheis and Kihl, 1995) samples both wet and dry dust deposition. Briefly, the dust trap consists of a Teflon- or enamel-coated angel-food cake pan mounted on a steel post about $2 \mathrm{~m}$ above the ground. This height nearly eliminates trapping of coarse grains traveling by saltation. Glass marbles fill the upper part of the pan above a supporting piece of 1/4-inch-mesh metal screen that rests $3-4 \mathrm{~cm}$ below the rim. The marbles simulate the effect of a gravelly fan surface and prevent dust that has filtered or washed into the bottom of the pan from being resuspended. To prevent birds from roosting, dust traps are fitted with two metal straps looped in an inverted basket shape over the pan, and the top surfaces of the straps are coated with Tanglefoot Bird Repellent. Samples are retrieved by washing the trap components with distilled water into plastic 1-L bottles. In the laboratory, a dust sample was dried at about $35^{\circ} \mathrm{C}$ in large evaporating dishes, and coarse organic material was removed. Total carbon and inorganic carbon were analyzed using a coulometer, and organic carbon content was calculated by difference. Soluble salt content was approximated by using measurements of electrical conductivity.

\section{Acknowledgments}

Reheis thanks the U.S. Geological Survey field assistants who helped with augering and sampling-Lanya Ross, Dhiren Khona, and Emily Edson. We also appreciate the numerous private landowners who courteously permitted access to their property. Eric Fisher performed laboratory analyses of dust samples, and Kelly Conrad prepared ostracode samples for identification.

\section{Literature Cited}

Laabs, B.J.C., 2001, Quaternary lake-level history and tectonic geomorphology in Bear Lake Valley, Utah and Idaho: Northern Arizona University, Flagstaff, M.S. thesis, 132 p.

Laabs, B.J.C., and Kaufman, D.S., 2003, Quaternary highstands in Bear Lake Valley, Utah and Idaho: Geological Society of America Bulletin, v. 115, p. 463-478.

Reheis, M.C., and Kihl, Rolf, 1995, Dust deposition in southern Nevada and California, 1984-1989: Relations to climate, source area, and source lithology: Journal of Geophysical Research, v. 100, no. D5, p. 8893-8918.

\section{Figure Captions}

Figure 1. Map of Bear Lake Valley showing site locations and generalized traces of Quaternary faults and modern and abandoned stream courses. Site numbers beginning with year (for example, 99BL-35) were described and sampled by Reheis; prefixes for some sites were omitted for ease of drafting, but all the omitted prefixes are for sites described in 1998 (for example, site 98BL-47 is abbreviated to 47). Site numbers beginning with BL- were described and sampled by Laabs; those beginning with DK- were described and sampled by Kaufman. A, airport; B, Bloomington; D, Dingle; GC, Garden City; M, Montpelier; P, Paris; SC, St. Charles.

Figure 2. Stratigraphic sections measured by Reheis from outcrops and auger holes in Bear Lake valley. Site locations are on figure 1 and table 1 . Sections are shown in approximate order from north to south on the following four pages. Column to 
right of lithology gives descriptive information such as color (informally described in field, not Munsell), sample data, and soil horizons. On some sections, brackets group depositional layers into different types of deposits (for example, loess) as interpreted from sedimentary characteristics.

Figure 3. Stratigraphic sections of exposures along the Rainbow Canal (see table 1). Most sections are outcrops, and correlation lines are physically traced, except that BL99-49 is an auger hole within a channel fill cut into older sediment, and correlations are uncertain. Surface altitudes were measured using a Trimble GPS and differentially corrected in the office. The base of the outcrop sections is the water level, thus the section bases essentially reflect the water gradient in the canal over a 2-day period when flow rate in the canal remained relatively constant. Measured sections then were plotted and their altitudes slightly adjusted to yield a smoothly sloping water level at the base of the outcrop sections. 
Table 1. Description of measured sections, auger holes, and sediment samples from the Bear Lake drainage basin, $1998-2001$.

[m: meters; $\mathbf{c m : ~ c e n t i m e t e r s ] ~}$

\begin{tabular}{|c|c|c|c|c|c|c|c|}
\hline $\begin{array}{l}\text { Sample } \\
\text { Number }\end{array}$ & General location & Latitude & Longitude & $\begin{array}{l}\text { Surface } \\
\text { altitude } \\
(\mathrm{m})\end{array}$ & $\begin{array}{l}\text { Description of unit } \\
\text { (depth below top of section, in } \mathrm{cm} \text { except as noted) }\end{array}$ & $\begin{array}{l}\text { Sample } \\
\text { depth }(\mathrm{cm})\end{array}$ & $\begin{array}{l}\text { Split for } \\
\text { ostracodes? } \\
\text { (see table 3) }\end{array}$ \\
\hline 98BL-1 & $\begin{array}{l}\text { Auger hole } \mathrm{W} \text { of Poleline } \\
\text { Road, } \mathrm{S} \text { of Bloomington } \\
\text { Creek, in small graben }\end{array}$ & $\begin{array}{l}\sim 42^{\circ} 09.4^{\prime} \\
\text { from map }\end{array}$ & $\begin{array}{l}\sim 111^{\circ} 22.1^{\prime} \\
\text { from map }\end{array}$ & $\begin{array}{l}\sim 1805.5 \text { from } \\
\text { map }\end{array}$ & $\begin{array}{l}\text { 0-10: Organic-rich muck } \\
\text { 10-110: Light-gray marl, becoming oxidized downward }\end{array}$ & & \\
\hline 98BL-2 & $\begin{array}{l}\text { Auger hole } \mathrm{W} \text { of Poleline } \\
\text { Road, on upthrown side of } \\
\text { graben at BL-1 }\end{array}$ & $\begin{array}{l}\sim 42^{\circ} 09.4^{\prime} \\
\text { from map }\end{array}$ & $\begin{array}{l}\sim 111^{\circ} 22.0^{\prime} \\
\text { from map }\end{array}$ & $\begin{array}{l}\sim 1807.5 \text { from } \\
\text { map }\end{array}$ & $\begin{array}{l}\text { 0- 40: Brown silt, plow zone plus subsoil } \\
\text { 40-110: Greenish-tan marl } \\
\text { 110-130+: Oxidized marl, sandy at base }\end{array}$ & & \\
\hline 98BL-5 & $\begin{array}{l}\text { Waterline trench for house, } \\
\text { W of Bear River }\end{array}$ & $\begin{array}{l}\sim 42^{\circ} 24.1^{\prime} \\
\text { from map }\end{array}$ & $\begin{array}{l}\sim 111^{\circ} 20.7^{\prime} \\
\text { from map }\end{array}$ & $\begin{array}{l}\sim 1830 \\
\text { from map }\end{array}$ & $\begin{array}{l}\text { 0-20: Ap (surface soil formed in loess) } \\
\text { 20-40: B1t } \\
\text { 40-88: B2t } \\
\text { 88-106: B3tk } \\
\text { 106-132: Bk } \\
\text { 132-152: 2Btk1b (paleosol formed in marl) } \\
\text { 152-192: 2Btk2b } \\
\text { 192-215: 2Cb (slightly oxidized marl) }\end{array}$ & $\begin{array}{l}0-20 \\
20-40 \\
40-88 \\
88-106 \\
106-132 \\
132-152 \\
152-192 \\
192-215\end{array}$ & $X$ \\
\hline 98BL-10A & $\begin{array}{l}\text { Sinkholes, S and E of Ovid. } \\
\text { Auger hole in center of } \\
\text { depression }\end{array}$ & $\begin{array}{l}\sim 42^{\circ} 17.5^{\prime} \\
\text { from map }\end{array}$ & $\begin{array}{r}\sim 111^{\circ} 25.9^{\prime} \\
\text { from map }\end{array}$ & $\begin{array}{l}\sim 1813.5 \text { from } \\
\text { map }\end{array}$ & $\begin{array}{l}\text { 0-30: Gray silt and clay (non-calcareous throughout) } \\
\text { 30-80: Oxidized silt and clay, Bw? } \\
\text { 80-210: Sand, silt, clay, a few pebbles; carbon present as plant } \\
\text { remains; mottled with Mn nodules below } 125 \mathrm{~cm} \\
\text { 210-245: Massive reddish clay }\end{array}$ & 85,105 & \\
\hline 98BL-10B & $\begin{array}{l}\text { Sinkholes, S and E of Ovid. } \\
\text { Auger hole on SE rim of } \\
\text { depression }\end{array}$ & $\begin{array}{l}\sim 42^{\circ} 17.5^{\prime} \\
\text { from map }\end{array}$ & $\begin{array}{l}\sim 111^{\circ} 25.9^{\prime} \\
\text { from map }\end{array}$ & $\begin{array}{l}\sim 1812 \\
\text { from map }\end{array}$ & $\begin{array}{l}\text { 0-120: Calcareous tan silt and clay } \\
\text { 120-150: Oxidized sand and silt, carbonate nodules } \\
\text { 150+: Rocky layer, possibly gravel or large carbonate nodules }\end{array}$ & & \\
\hline 98BL-11 & $\begin{array}{l}\text { Dingle scarp, } S \text { of Dingle, } \\
\text { roadcut next to old barn }\end{array}$ & $\begin{array}{l}\sim 42^{\circ} 12.1^{\prime} \\
\text { from map }\end{array}$ & $\begin{array}{l}\sim 111^{\circ} 16.0^{\prime} \\
\text { from map }\end{array}$ & $\begin{array}{l}\sim 1818 \\
\text { from map }\end{array}$ & $\begin{array}{l}\text { 0-150: Loess, } \mathrm{Bw} / \mathrm{Bk} \text {, stage } \mathrm{II} \mathrm{CaCO}_{3} \text {. Cicada burrows. } \\
\text { 150-250+: Reddish fine sand over river gravel. Weak paleosol } \\
(\mathrm{Bwj} / \mathrm{Bk}) \text { in sand, stage }{\mathrm{II} \mathrm{CaCO}_{3}}\end{array}$ & & \\
\hline 99BL-26 & $\begin{array}{l}\text { Rainbow Canal } \mathrm{N} \text { of airport } \\
\text { road crossing, at minor } \\
\text { channel }\end{array}$ & $42^{\circ} 13.992^{\prime}$ & $111^{\circ} 17.821^{\prime}$ & 1810.37 & $\begin{array}{l}0-145 \text { : Moderately sorted sand, silt, and pebbles in two channel } \\
\text { fills, } 0-80 \text { and } 80-145 \mathrm{~cm} \text {. Underlain by well-washed, } \\
\text { crossbedded sand with abundant bivalves and snails to } 200 \mathrm{~cm} \text {, in } \\
\text { turn by gravel to water at } 310 \mathrm{~cm}\end{array}$ & $\begin{array}{l}\mathrm{B}, 80-100 \\
\mathrm{~A}, 170-200\end{array}$ & \\
\hline 99BL-31 & $\begin{array}{l}\text { Auger hole S of Bloomington } \\
\text { Creek }\end{array}$ & $42^{\circ} 09.9483^{\prime}$ & $111^{\circ} 22.1540^{\prime}$ & 1809.00 & $\begin{array}{l}\text { 0-10: Organic-rich silty clay } \\
\text { 10-60: Bk in marl } \\
\text { 60-150: Carbonate nodules in marl; oxidation below } 120 \mathrm{~cm} \\
\text { 150-270: Interbedded fine, well-sorted sand and silty clay; } \\
\text { probably lacustrine }\end{array}$ & & \\
\hline
\end{tabular}


Table 1. Description of measured sections, auger holes, and sediment samples from the Bear Lake drainage basin, 1998-2001-Continued.

\begin{tabular}{|c|c|c|c|c|c|c|c|}
\hline $\begin{array}{l}\text { Sample } \\
\text { Number }^{1}\end{array}$ & General location & Latitude & Longitude & $\begin{array}{l}\text { Surface } \\
\text { altitude } \\
(\mathrm{m})\end{array}$ & $\begin{array}{l}\text { Description of unit } \\
\text { (depth below top of section, in cm except as noted) }\end{array}$ & $\begin{array}{l}\text { Sample } \\
\text { depth }(\mathrm{cm})\end{array}$ & $\begin{array}{l}\text { Split for } \\
\text { ostracodes? } \\
\text { (see table 3) }\end{array}$ \\
\hline 99BL-32 & $\begin{array}{l}\text { Auger hole to NW of 99BL- } \\
31 \text { on same geomorphic } \\
\text { feature }\end{array}$ & $\begin{array}{l}\sim 42^{\circ} 10.0^{\prime} \\
\text { from map }\end{array}$ & $\begin{array}{r}\sim 111^{\circ} 22.5^{\prime} \\
\text { from map }\end{array}$ & $\begin{array}{l}\sim 1808 \\
\text { from map }\end{array}$ & $\begin{array}{l}\text { 0-60: Organic-rich soil formed in silt loam and fine sand; levee } \\
\text { deposits? } \\
\text { 60-80: Organic-rich A horizon formed in marl and fine sand } \\
\text { 80-120: Fine silty sand } \\
\text { 120-155: Marl }\end{array}$ & & \\
\hline 99BL-33 & $\begin{array}{l}\text { Auger hole } \mathrm{N} \text { of St. Charles. } \\
\text { Large abandoned channel }\end{array}$ & $42^{\circ} 08.8632^{\prime}$ & $111^{\circ} 23.3393^{\prime}$ & 1810.00 & $\begin{array}{l}0-10: \text { Organic-rich layer } \\
\text { 10-70: Marl } \\
\text { 70-80: Silty sand } \\
\text { 80-150+: Marl }\end{array}$ & & \\
\hline 99BL-34 & $\begin{array}{l}\text { Auger hole, east of } \\
\text { Bloomington scarp }\end{array}$ & $42^{\circ} 11.0637^{\prime}$ & $111^{\circ} 22.6253^{\prime}$ & 1809.00 & $\begin{array}{l}\text { 0-50: Organic-rich silty clay with snails } \\
\text { 50-110: Marly silty clay; shell fragments } \\
\text { 110-200+: Slightly calcareous mottled orange/gray clay }\end{array}$ & $\begin{array}{l}0-50 \\
100-120\end{array}$ & \\
\hline 99BL-35 & Old railroad cut NE of Paris & $42^{\circ} 14.2122^{\prime}$ & $111^{\circ} 23.5704^{\prime}$ & 1815.00 & $\begin{array}{l}\text { 0-95: Pale sandy silt (loess?), cicada burrows; } 20 \mathrm{~cm} \mathrm{~A} \mathrm{on} \mathrm{Bk} \\
\text { 95-245: Weak paleosol (Bw) in massive silt and sand; bedded fine } \\
\text { sand at base } \\
\text { 245-280: Paleosol in silt and clay: Organic-rich A, dark Bt } \\
\text { 280-340: Btk, less strong structure } \\
\text { 340-460: Oxidized, bedded calcareous marl; interbedded with } \\
\text { sand at base } \\
\text { 460-530+: Well-bedded, fine-medium reddish lacustrine sand }\end{array}$ & $\begin{array}{l}390-410 \\
430-450 \\
480-510\end{array}$ & $\begin{array}{l}X \\
X\end{array}$ \\
\hline 99BL-36 & $\begin{array}{l}\text { Auger hole on refuge road, } \mathrm{E} \\
\text { of Bloomington, down side } \\
\text { of fault cutting meander }\end{array}$ & $42^{\circ} 12.6589^{\prime}$ & $111^{\circ} 21.7521^{\prime}$ & 1808.00 & $\begin{array}{l}\text { 0-20: Organic-rich calcareous clay } \\
\text { 20-80: Marl; oxidized below } 60 \\
\text { 80-170: Calcareous reddish silt and v. fine sand, well sorted } \\
\text { 170-240: Reddish fine sand }\end{array}$ & & \\
\hline 99BL-37 & $\begin{array}{l}\text { Auger hole E of } \\
\text { Bloomington, up side of } \\
\text { fault at 99BL-36 }\end{array}$ & $42^{\circ} 12.7752^{\prime}$ & $111^{\circ} 21.8142^{\prime}$ & $\begin{array}{l}1810.00 \\
\text { (at base of } \\
\text { cut) }\end{array}$ & $\begin{array}{l}\text { 0-80: Organic-rich clay } \\
\text { 80-200: Calcareous silt and fine sand, orange mottled, shelly } \\
\text { 200-230+: Reddish well-sorted fine sand, with shells }\end{array}$ & $\begin{array}{l}70-80 \\
80-100 \\
200-230\end{array}$ & $\mathrm{X}$ \\
\hline 99BL-38 & $\begin{array}{l}\text { Auger hole on N side Paris- } \\
\text { Dingle road, W of outlet } \\
\text { canal, abandoned channel of } \\
\text { Bear River }\end{array}$ & $42^{\circ} 14.1401^{\prime}$ & $111^{\circ} 21.6109^{\prime}$ & 1807.92 & $\begin{array}{l}0-20 \text { : Organic-rich silty clay } \\
\text { 20-85: Calcareous silty clay, upper part is Bk horizon } \\
\text { 85-180: Oxidized slightly calcareous clay, silt, and sand } \\
\text { 230-280+: Gray shelly medium-coarse sand }\end{array}$ & $230-250$ & \\
\hline 99BL-39 & $\begin{array}{l}\text { Auger hole } \mathrm{E} \text { of airport, } \mathrm{N} \\
\text { side, abandoned channel of } \\
\text { Bear River }\end{array}$ & $42^{\circ} 15.1191^{\prime}$ & $111^{\circ} 19.4917^{\prime}$ & 1808.73 & $\begin{array}{l}\text { 0-50: Organic-rich A }(10 \mathrm{~cm}) \text { overlies pink silty clay } \\
\text { 50-70: Black, organic-rich shelly mud. Grades down into } \\
\text { 70-175: Reddish sand, coarsening downward }\end{array}$ & $\begin{array}{l}60-70 \\
130-175\end{array}$ & \\
\hline
\end{tabular}


Table 1. Description of measured sections, auger holes, and sediment samples from the Bear Lake drainage basin, 1998-2001-Continued.

\begin{tabular}{|c|c|c|c|c|c|c|c|}
\hline $\begin{array}{l}\text { Sample } \\
\text { Number }\end{array}$ & General location & Latitude & Longitude & $\begin{array}{l}\text { Surface } \\
\text { altitude } \\
(\mathrm{m})\end{array}$ & $\begin{array}{l}\text { Description of unit } \\
\text { (depth below top of section, in cm except as noted) }\end{array}$ & $\begin{array}{l}\text { Sample } \\
\text { depth }(\mathrm{cm})\end{array}$ & $\begin{array}{l}\text { Split for } \\
\text { ostracodes? } \\
\text { (see table 3) }\end{array}$ \\
\hline 99BL-40 & $\begin{array}{l}\text { Auger hole E of airport, } \mathrm{S} \\
\text { side, abandoned channel of } \\
\text { Bear River }\end{array}$ & $42^{\circ} 14.1014^{\prime}$ & $111^{\circ} 19.5588^{\prime}$ & 1809.69 & $\begin{array}{l}\text { 0-10: Spoil } \\
\text { 10-30: Organic-rich sandy mud } \\
\text { 30-80: Bk horizon, mud } \\
\text { 80-130: Marly mud } \\
\text { 130-220: Reddish calcareous medium sand }\end{array}$ & & \\
\hline 99BL-41 & $\begin{array}{l}\text { Outlet canal cut and auger, } \mathrm{S} \\
\text { of bridge on airport road; } \\
\text { lake plain }\end{array}$ & $\begin{array}{l}\sim 42^{\circ} 14.1^{\prime} \\
\text { from map }\end{array}$ & $\begin{array}{l}111^{\circ} 21.5^{\prime} \\
\text { from map }\end{array}$ & $\begin{array}{l}1804.4 \\
\text { from map }\end{array}$ & $\begin{array}{l}\text { 130-220: Reddish calcareous medium sand } \\
0-20 \text { : Organic-rich shelly mud } \\
\text { 20-110: Bk grading down into reddish marly clay } \\
\text { 110-270: Reddish marly clay (augered below 140) }\end{array}$ & $160-215$ & \\
\hline 99BL-42 & $\begin{array}{l}\text { Auger hole, W of outlet } \\
\text { canal, N of Mud Lake dam; } \\
\text { Bear River or Bear Lake } \\
\text { channel? }\end{array}$ & $42^{\circ} 12.8604^{\prime}$ & $111^{\circ} 20.5828^{\prime}$ & 1807.51 & $\begin{array}{l}0-25: \text { Organic-rich mud w/ shell fragments } \\
\text { 25-125: Calcareous gray marl, oxidized toward base } \\
\text { 125-180: Reddish fine sand, a few shells } \\
\text { 180-225: Orange medium sand, shell fragments }\end{array}$ & $\begin{array}{l}0-25 \\
140-160 \\
200-220\end{array}$ & $X$ \\
\hline 99BL-43 & $\begin{array}{l}\text { Auger hole, E of outlet canal, } \\
\mathrm{N} \text { of airport; lake plain- } \\
\text { delta top? }\end{array}$ & $42^{\circ} 17.1812^{\prime}$ & $111^{\circ} 21.0170^{\prime}$ & 1807.59 & $\begin{array}{l}\text { 0-60: Silty sand, gray at top, soil structure and color increases } \\
\text { downward } \\
\text { 60-145: Reddish silt, clay, and fine sand } \\
\text { 145-270: Interbedded? muddy medium and fine sand, reddish, } \\
\text { slightly to non-calcareous, monotonous }\end{array}$ & $\begin{array}{l}155-180 \\
240-270\end{array}$ & $\begin{array}{l}X \\
X\end{array}$ \\
\hline 99BL-44 & $\begin{array}{l}\text { Cut, W side Rainbow Canal, } \\
\mathrm{N} \text { end canal; loess over river } \\
\text { (delta) gravel? }\end{array}$ & $42^{\circ} 14.4883^{\prime}$ & $111^{\circ} 17.5449^{\prime}$ & 1810.61 & $\begin{array}{l}\text { 0-105: Loess or marl, A/Bk (stage II); carbonate groundwater } \\
\text { nodules at base } \\
\text { 105-150: Alluvial sand, but bedding not obvious; obliterated by } \\
\text { surface soil } \\
\text { 150-250: (canal level) Pebble-cobble gravel }\end{array}$ & $120-140$ & \\
\hline 99BL-45 & $\begin{array}{l}\text { Cut, W side Rainbow Canal. } \\
\text { Abandoned channel of Bear } \\
\text { River, inset into deposits } \\
\text { exposed at 99BL-44 }\end{array}$ & $42^{\circ} 14.2702^{\prime}$ & $111^{\circ} 17.6390^{\prime}$ & 1810.28 & $\begin{array}{l}\text { 0-60: Light gray mud, finely laminated at base, little or no soil } \\
\text { formation, rare snails } \\
\text { 60-130: Calcareous, organic-rich silt and clay, abundant snails } \\
\text { throughout. [n.b.: Entire unit 0-220+ is cut-and-fill into older } \\
\text { loess over gravel as at 99BL-44] } \\
\text { 130-200: Orange medium sand (sharp upper contact) } \\
\text { 200-220+: Sandy pebble gravel w/ common clam shells }\end{array}$ & $\begin{array}{l}60-65 \\
120-130 \\
200-220\end{array}$ & $X$ \\
\hline 99BL-46 & $\begin{array}{l}\text { Cut, W side Rainbow Canal. } \\
\text { Abandoned channel of Bear } \\
\text { River, inset into river gravel }\end{array}$ & $42^{\circ} 13.5771^{\prime}$ & $111^{\circ} 17.7503^{\prime}$ & 1810.74 & $\begin{array}{l}\text { 0-110: Alluvium w/ minor pebble beds; abundant shells } \\
\text { 110-250+: Pebble to cobble gravel w/ clam shells. Locally a } \\
\text { cicada-burrowed tan silt preserved at upper contact, presumed } \\
\text { same as loess at 99BL-44 }\end{array}$ & $\begin{array}{l}60-90 \\
110-140\end{array}$ & \\
\hline 99BL-47A & $\begin{array}{l}\text { Cut, W side Rainbow Canal, } \\
\text { S of airport road. Gradual } \\
\text { burial of sediments at } \\
\text { 99BL-25 }\end{array}$ & $42^{\circ} 13.0992^{\prime}$ & $111^{\circ} 17.7060^{\prime}$ & 1810.13 & $\begin{array}{l}\text { 0-60: Accretionary floodplain and marsh deposits } \\
\text { 60-85: Grayish-black organic-rich layer } \\
\text { 85-250: Bk formed in calcareous reddish marl; coarsens } \\
\text { downward into silty fine sand } \\
\text { 250-260+: Pebble to cobble gravel w/clam shells }\end{array}$ & & \\
\hline
\end{tabular}


Table 1. Description of measured sections, auger holes, and sediment samples from the Bear Lake drainage basin, 1998-2001-Continued.

\begin{tabular}{|c|c|c|c|c|c|c|c|}
\hline $\begin{array}{l}\text { Sample } \\
\text { Number }^{1}\end{array}$ & General location & Latitude & Longitude & $\begin{array}{l}\text { Surface } \\
\text { altitude } \\
(\mathrm{m})\end{array}$ & $\begin{array}{l}\text { Description of unit } \\
\text { (depth below top of section, in } \mathrm{cm} \text { except as noted) }\end{array}$ & $\begin{array}{l}\text { Sample } \\
\text { depth }(\mathrm{cm})\end{array}$ & $\begin{array}{l}\text { Split for } \\
\text { ostracodes? } \\
\text { (see table 3) }\end{array}$ \\
\hline 99BL-47 & $\begin{array}{l}\text { Cut, W side Rainbow Canal, } \\
\text { S of airport road. Gradual } \\
\text { burial of sediments at } \\
\text { 99BL-25 }\end{array}$ & $42^{\circ} 13.0010^{\prime}$ & $111^{\circ} 17.7616^{\prime}$ & 1810.52 & $\begin{array}{l}\text { 0-85: Accretionary floodplain and marsh deposits } \\
\text { 90-110: Grayish-black organic-rich layer } \\
\text { 110-200: Bk formed in calcareous reddish marl; coarsens } \\
\text { downward into silty fine sand at least } 40 \mathrm{~cm} \text { thick }\end{array}$ & $0-80$ & \\
\hline 99BL-48A & $\begin{array}{l}\text { Cut, W side Rainbow Canal, } \\
\text { S of airport road, a few } \\
\text { meters up-canal from corral } \\
\text { gate }\end{array}$ & $42^{\circ} 12.6575^{\prime}$ & $11117^{\circ} 7048^{\prime}$ & 1808.795 & $\begin{array}{l}\text { Green clay, locally oxidized, below } 165 \mathrm{~cm} \text { to water level; } \\
\text { overlain by calcareous pink marl and in turn by black organic-rich } \\
\text { layer at } 110-115 \mathrm{~cm} \text {; in turn by accretion sediment }\end{array}$ & $165-185$ & $X$ \\
\hline 99BL-48B & $\begin{array}{l}\text { Cut, W side Rainbow Canal, } \\
\mathrm{S} \text { of airport road. Black } \\
\text { layer becomes multiple } \\
\text { black layers }\end{array}$ & $42^{\circ} 12.6540^{\prime}$ & $111^{\circ} 17.7060^{\prime}$ & 1809.99 & $\begin{array}{l}\text { Bands of accretionary sediment, finer grained than to north, with } \\
\text { interbedded thin black layers overlying marly deposits }\end{array}$ & $110-140$ & \\
\hline \multirow[t]{3}{*}{ 99BL-49 } & \multirow[t]{3}{*}{$\begin{array}{l}\text { Auger hole, E side Rainbow } \\
\text { Canal at flume. Abandoned } \\
\text { channel of Bear River }\end{array}$} & \multirow[t]{3}{*}{$42^{\circ} 12.4719^{\prime}$} & \multirow[t]{3}{*}{$111^{\circ} 17.7213^{\prime}$} & \multirow[t]{3}{*}{1808.97} & $\begin{array}{l}0-55: \text { Organic-rich silt and clay; rare shells } \\
\text { 55-100: Marly silt and clay } \\
\text { 100-175: Reddish mud, slightly calcareous }\end{array}$ & $20-50$ & \\
\hline & & & & & $\begin{array}{l}\text { 175-270: Reddish-gray silt and sand, abundant plant fragments, } \\
\text { slightly calcareous }\end{array}$ & $190-215$ & $X$ \\
\hline & & & & & $\begin{array}{l}\text { 270-320: Gray silty sand, slightly calcareous, shell fragments } \\
\text { 320+: Gravel (did not penetrate) }\end{array}$ & $270-300$ & $\mathrm{X}$ \\
\hline 99BL-53 & $\begin{array}{l}\text { NE side of US-30 roadcut, } \\
\text { SE corner Montpelier Quad }\end{array}$ & $42^{\circ} 15.5642^{\prime}$ & $111^{\circ} 15.8441^{\prime}$ & $\begin{array}{l}1867.69 \\
\text { (top of } \\
\text { sand beds) }\end{array}$ & $\begin{array}{l}\sim 20 \mathrm{~m} \text { of dominantly well-sorted well-bedded fine-med. sand. } \\
\text { Capped by terrace gravels. } 5 \mathrm{~m} \text { above base are green clay beds } \\
\text { (location of A and paleomagnetic samples) }\end{array}$ & $\begin{array}{l}\sim 10 \mathrm{~m}(\mathrm{~B}) \\
\sim 15 \mathrm{~m}(\mathrm{~A})\end{array}$ & $\begin{array}{l}X \\
X\end{array}$ \\
\hline 99BL-59 & $\begin{array}{l}\text { Drainage-ditch cut, along } \\
\text { fault } S \text { of Dingle, E side } \\
\text { road }\end{array}$ & $42^{\circ} 11.6398^{\prime}$ & $111^{\circ} 16.0818^{\prime}$ & 1820.5 & $\begin{array}{l}\text { Colluvium overlies lacustrine deposits extending from GPS point } \\
\text { at road level, } 2 \text { m below road and } \sim 4 \mathrm{~m} \text { above. Interbedded lake } \\
\text { gravel, sand, and mud. } 2 \text { beds of shells near base. Lower is } \\
\text { scattered snail shells in mud; upper ( } 1 \mathrm{~m} \text { higher), at top of silty } \\
\text { sand, is shell hash }\end{array}$ & $\begin{array}{l}\text { upper (B) } \\
\text { lower (A) }\end{array}$ & \\
\hline 00BL-8 & $\begin{array}{l}\text { W of Garden City at edge of } \\
\text { raspberry patch }\end{array}$ & $41^{\circ} 56.3445^{\prime}$ & $111^{\circ} 24.2478^{\prime}$ & 1824.9 & $\begin{array}{l}\text { Orange calcareous mud with black organic remains and sticks } \\
\text { present at } \sim 200 \mathrm{~cm} \text {, dated at } 320 \pm 40{ }^{14} \mathrm{C} \text { yr. Massive, silt-rich. } \\
\text { Grades down into mottled mud, less organics, contains thin } \mathrm{f}-\mathrm{m} \\
\text { sand lenses }\end{array}$ & $\begin{array}{l}151-162 \\
235-333\end{array}$ & $X$ \\
\hline $\begin{array}{l}\text { 00BL-12 } \\
\text { (not in fig. } \\
1 \text { ) }\end{array}$ & $\begin{array}{l}\text { E-central edge Meadowville } \\
\text { quad at irrigation ditch } \\
\text { blowout }\end{array}$ & $\begin{array}{l}\sim 41^{\circ} 49.1^{\prime} \\
\text { from map }\end{array}$ & $\begin{array}{r}\sim 111^{\circ} 22.9^{\prime} \\
\text { from map }\end{array}$ & $\begin{array}{l}\sim 1823 \\
\text { from map }\end{array}$ & $\begin{array}{l}\text { Pale pinkish fine-grained deposits, deeply weathered, about } 3 \mathrm{~m} \\
\text { thick, right on } 6000 \text {-foot contour. Probable loess. Sampled at } \\
\text { base of exposure }\end{array}$ & & \\
\hline 00BL-27 & $\begin{array}{l}\text { Outlet canal S of US-89, W- } \\
\text { central Montpelier quad }\end{array}$ & $\begin{array}{l}\sim 42^{\circ} 30.0^{\prime} \\
\text { from map }\end{array}$ & $\begin{array}{l}\sim 111^{\circ} 21.5^{\prime} \\
\text { from map }\end{array}$ & $\begin{array}{l}\sim 1804 \\
\text { from map }\end{array}$ & $\begin{array}{l}\text { Minor channel fill inset into organic-rich sediment interbedded } \\
\text { with pale white marly layers. Sandy channel fill has abundant } \\
\text { snails }\end{array}$ & $10-20$ & \\
\hline
\end{tabular}


Table 1. Description of measured sections, auger holes, and sediment samples from the Bear Lake drainage basin, 1998-2001-Continued.

\begin{tabular}{|c|c|c|c|c|c|c|c|}
\hline $\begin{array}{l}\text { Sample } \\
\text { Number }\end{array}$ & General location & Latitude & Longitude & $\begin{array}{l}\text { Surface } \\
\text { altitude } \\
(\mathrm{m})\end{array}$ & $\begin{array}{l}\text { Description of unit } \\
\text { (depth below top of section, in } \mathrm{cm} \text { except as noted) }\end{array}$ & $\begin{array}{l}\text { Sample } \\
\text { depth }(\mathrm{cm})\end{array}$ & $\begin{array}{l}\text { Split for } \\
\text { ostracodes? } \\
\text { (see table 3) }\end{array}$ \\
\hline $\begin{array}{l}\text { 00BL-29 } \\
\text { (not in fig. } \\
1 \text { ) }\end{array}$ & $\begin{array}{l}\text { Roadcut S of Boy Scout } \\
\text { Aquatics Camp, E side Bear } \\
\text { Lake }\end{array}$ & $41^{\circ} 53.3940^{\prime}$ & $111^{\circ} 15.7980^{\prime}$ & 1835.7 & $\begin{array}{l}\text { Highest point of well-rounded beach clasts in small fan-delta. } \\
\text { Overlain by } 1-2 \mathrm{~m} \text { of angular fan gravel and in turn by red fine- } \\
\text { grained alluvium. Surface soil has Btjk horizon with stage I } \\
\mathrm{CaCO}_{3}\end{array}$ & & \\
\hline 00BL-54 & $\begin{array}{l}\text { Powerline roadcut } \mathrm{N} \text { of } \\
\text { Indian Creek, NE corner } \\
\text { Bear Lake } \mathrm{N} \text { quad }\end{array}$ & $42^{\circ} 06.4185^{\prime}$ & $111^{\circ} 15.6659^{\prime}$ & $\begin{array}{l}1851.7 \text { (base) } \\
1866.3 \\
\text { (top) }\end{array}$ & $\begin{array}{l}\text { Sequence of lacustrine deposits } \sim 22 \mathrm{~m} \text { thick intercalated with fan } \\
\text { gravel. Base is beach gravel interbedded with silty sand (A), } \\
\text { fining upward into brown silt and mudstone (B), gray mdst. (C), } \\
\text { pale gray fine silty sand (D), pale gray silty mdst. (E), and reddish } \\
\text { mdst., coarsening upward into fan gravel. Measurements are } \\
\text { meters above base of lacustrine deposits }\end{array}$ & $\begin{array}{l}\text { E, } 6.8 \mathrm{~m} \\
\mathrm{D}, 9.2 \mathrm{~m} \\
\mathrm{C}, 10.4 \mathrm{~m} \\
\mathrm{~B}, 13.2 \mathrm{~m} \\
\mathrm{~A}, 19.0 \mathrm{~m}\end{array}$ & $\begin{array}{l}X \\
X \\
X \\
X \\
X\end{array}$ \\
\hline 00BL-59 & $\begin{array}{l}\text { Faulted fans and beach } \\
\text { gravel N of Indian Creek, } \\
\text { Bear Lake N quad }\end{array}$ & $42^{\circ} 06.2895^{\prime}$ & $111^{\circ} 15.6281^{\prime}$ & 1852.0 & $\begin{array}{l}\text { Upper of two beach gravels overlain by a thin shell-bearing } \\
\text { reddish mudstone capped by fan gravel. Beach gravels are cut by } \\
\text { several faults }\end{array}$ & & \\
\hline 00BL-60 & $\begin{array}{l}\text { Landslide outcrop above } \\
\text { highway just } \mathrm{N} \text { of Indian } \\
\text { Creek, NE corner Bear Lake } \\
\mathrm{N} \text { quad }\end{array}$ & $42^{\circ} 05.7446$ & $111^{\circ} 15.4779^{\prime}$ & 1846.3 & $\begin{array}{l}\text { Lacustrine deposits overlain by fan gravel. Basal part is olive- } \\
\text { green clay (A), overlain by several meters of gray sand (B), and } \\
\text { in turn by reddish mudstone }(\mathrm{C}) \text { near top of cut. At } \mathrm{S} \text { edge of cut, } \\
\text { a thin beach gravel lies between the reddish mudstone and } \\
\text { overlying fan gravel }\end{array}$ & $\begin{array}{l}\text { C } \\
\text { B } \\
\text { A }\end{array}$ & $\begin{array}{l}X \\
X \\
X\end{array}$ \\
\hline 00BL-61 & $\begin{array}{l}\text { East of highway above } \\
\text { 00BL-60 (above landslide), } \\
\text { NE corner Bear Lake N } \\
\text { quad }\end{array}$ & $42^{\circ} 05.8253^{\prime}$ & $111^{\circ} 15.3732^{\prime}$ & 1869.7 & $\begin{array}{l}\text { Similar to 00BL-60 but much higher in elevation and lacking basal } \\
\text { green clay. Possibly same lake unit as 00BL-60, separated by } \\
\text { fault. Gray sand (A) at base of lake deposits overlain by reddish } \\
\text { mudstone (B) capped by fan gravel }\end{array}$ & $\begin{array}{l}\mathrm{B} \\
\mathrm{A}\end{array}$ & $\begin{array}{l}X \\
X\end{array}$ \\
\hline 00BL-63 & $\begin{array}{l}\text { Waterline trench being } \\
\text { backfilled as I sampled. S of } \\
\text { Indian Creek, NE corner } \\
\text { Bear Lake N quad }\end{array}$ & $\begin{array}{l}42^{\circ} 05.1810^{\prime} \\
42^{\circ} 05.1811^{\prime}\end{array}$ & $\begin{array}{l}111^{\circ} 15.2927^{\prime} \\
111^{\circ} 15.3086^{\prime}\end{array}$ & $\begin{array}{l}1842.8 \\
\text { (top) } \\
1837.2 \\
\text { (near base) }\end{array}$ & $\begin{array}{l}\text { Lacustrine deposits interbedded with fan gravel. Sediments are } \\
\text { impregnated with gypsum crystals. Shells are abundant and beach } \\
\text { gravel crops out on slope to south. Upper part of trench cut in } \\
\text { spring-discharge deposits }\end{array}$ & $\begin{array}{l}\text { B (upper) } \\
\text { A (lower) }\end{array}$ & $\begin{array}{l}X \\
X\end{array}$ \\
\hline 00BL-64 & $\begin{array}{l}\text { Old borrow pit, } \mathrm{S} \text { of Indian } \\
\text { Creek, NE corner Bear Lake } \\
\mathrm{N} \text { quad }\end{array}$ & $\begin{array}{l}\sim 42^{\circ} 05.3 \\
\text { from map }\end{array}$ & $\begin{array}{l}\sim 111^{\circ} 15.4^{\prime} \\
\text { from map }\end{array}$ & $\begin{array}{l}\sim 1816.5 \text { from } \\
\text { map }\end{array}$ & $\begin{array}{l}\text { Beach gravel overlain by } 1 \mathrm{~m} \text { of massive red sandy mudstone, } \\
\text { overlain by } 15-20 \mathrm{~cm} \text { channel gravel containing shells, in turn by } \\
\text { weakly bedded alluvium }\end{array}$ & & \\
\hline 01BL-23 & $\begin{array}{l}\text { W side of small pond } \mathrm{N} \text { of } \\
\text { Merkley Lake, E-central } \\
\text { Dingle quad }\end{array}$ & $42^{\circ} 11.59^{\prime}$ & $111^{\circ} 16.38^{\prime}$ & $\begin{array}{l}\sim 1810.5 \text { from } \\
\text { map }\end{array}$ & $\begin{array}{l}\text { Lacustrine? or spring discharge deposits atop reddish Bear River } \\
\text { sand. Reddish fine sand (A) }>50 \mathrm{~cm} \text { thick overlain by } \sim 25 \mathrm{~cm} \text { of } \\
\text { calcareous pink marl (B). May be downfaulted lake-plain } \\
\text { deposits }\end{array}$ & $\begin{array}{l}\text { B ( }(37-44) \\
\text { A (sand) }\end{array}$ & $X$ \\
\hline
\end{tabular}


Table 1. Description of measured sections, auger holes, and sediment samples from the Bear Lake drainage basin, 1998-2001-Continued.

\begin{tabular}{|c|c|c|c|c|c|c|c|}
\hline $\begin{array}{l}\text { Sample } \\
\text { Number }^{1}\end{array}$ & General location & Latitude & Longitude & $\begin{array}{l}\text { Surface } \\
\text { altitude } \\
\text { (m) }\end{array}$ & $\begin{array}{l}\text { Description of unit } \\
\text { (depth below top of section, in cm except as noted) }\end{array}$ & $\begin{array}{l}\text { Sample } \\
\text { depth }(\mathrm{cm})\end{array}$ & $\begin{array}{l}\text { Split for } \\
\text { ostracodes? } \\
\text { (see table 3) }\end{array}$ \\
\hline 01BL-24 & $\begin{array}{l}\text { Roadcut E of road just S of } \\
\text { small pond at 01BL-23, E- } \\
\text { central Dingle quad, } \\
\text { footwall block }\end{array}$ & $\begin{array}{l}42^{\circ} 11.48^{\prime} \\
\text { (base of } \\
\text { section; } \\
\text { measured on } \\
\text { road) }\end{array}$ & $\begin{array}{l}111^{\circ} 16.05^{\prime} \\
\text { (base of } \\
\text { section; } \\
\text { measured on } \\
\text { road) }\end{array}$ & $\begin{array}{l}1820.5 \\
\text { (on road, } \\
\text { est. from } \\
\text { 99BL-59)- } \\
\text { top } 1841.9\end{array}$ & $\begin{array}{l}\text { 21.4-m measured section. Shoreface deposits interbedded with } \\
\text { fault-scarp colluvium and spring-discharge deposits. } \\
\text { Measurements begin } 10.5 \mathrm{~m} \text { above roadbed. A, nearshore sand } \\
\text { between beach gravel beds, rare shells; B, reddish silty clay, rare } \\
\text { shells, Bear River into lake?; C, mixed clay and gravel, spring + } \\
\text { colluvium?; D, similar to B }\end{array}$ & $\begin{array}{l}\text { D, } 100-320 \\
\text { C, } 320-420 \\
\text { B, 420-610 } \\
\text { A, } 660-730\end{array}$ & $\begin{array}{l}X \\
X \\
X\end{array}$ \\
\hline 01BL-30 & $\begin{array}{l}\text { Leach test hole just } \mathrm{S} \text { of road } \\
\text { to Bern cemetery, W of } \\
\text { highway, Ovid 7.5' quad }\end{array}$ & $42^{\circ} 19.81^{\prime}$ & $111^{\circ} 23.26^{\prime}$ & $\begin{array}{l}\sim 1820 \\
\text { from map }\end{array}$ & $\begin{array}{l}\text { Loess overlying lake and marsh deposits; entire section } \\
\text { noncalcareous. Surface soil to } \sim 1.45 \mathrm{~m} \text {; mollic A horizon over } \\
\text { argillic Bt; buried soil with argillic Bt to } 2.3 \mathrm{~m} \text {. A, pale olive- } \\
\text { green sandy silt, well bedded. B, silty fine sand to coarse sand, } \\
\text { unbedded. C, pale olive silt and sand, root traces; capped by } 2 \mathrm{~cm} \\
\text { of fine-medium, well-sorted sand }\end{array}$ & $\begin{array}{l}\text { C, } 233-250 \\
\text { B, 250-285 } \\
\text { A, } 285-300\end{array}$ & $X$ \\
\hline 01BL-34 & $\begin{array}{l}\text { Borrow pit W of US } 30 \text { at } \\
\text { mouth of Bear Hollow, SE } \\
\text { Montpelier quad }\end{array}$ & $\sim 42^{\circ} 16.5^{\prime}$ & $\sim 111^{\circ} 17^{\prime}$ & $\begin{array}{l}\sim 1815 \\
\text { from map }\end{array}$ & $\begin{array}{l}\text { Fan-delta of Bear Hollow drainage merging with Bear River } \\
\text { fluvial deposits. Sampled snails in sandy lens ca. } 3.5 \mathrm{~m} \text { below } \\
\text { surface on S side pit }\end{array}$ & 350 & \\
\hline 01BL-35 & $\begin{array}{l}\text { Bear River cutbank } \mathrm{N} \text { of US } \\
30 \text { and } \mathrm{E} \text { of outlet canal, W- } \\
\text { central Montpelier quad }\end{array}$ & $42^{\circ} 19.81^{\prime}$ & $111^{\circ} 23.26^{\prime}$ & $\begin{array}{l}\sim 1806 \\
\text { from map }\end{array}$ & $\begin{array}{l}\text { Marl, presumably lake-bottom sediment, with } \mathrm{A} / \mathrm{Btj} / \mathrm{Bk} / \mathrm{C} \text { profile. } \\
\text { Marl (sample B) overlies well-sorted, fine-medium crossbedded } \\
\text { sand (sample A), probably fluvial; contact may be erosional. } \\
\text { Snails in both samples }\end{array}$ & $\begin{array}{l}\text { B, } 150-190 \\
\text { A, } 190-250\end{array}$ & $X$ \\
\hline 01BL-39 & $\begin{array}{l}\text { Bear River cutbank WSW of } \\
\text { Bennington, down-stream of } \\
\text { railway bridge }\end{array}$ & $\begin{array}{l}\sim 42^{\circ} 22.9^{\prime} \\
\text { from map }\end{array}$ & $\begin{array}{l}\sim 111^{\circ} 20.7^{\prime} \\
\text { from map }\end{array}$ & $\begin{array}{l}\sim 1844 \\
\text { from map }\end{array}$ & $\begin{array}{l}\text { Bear River pebble-cobble gravel } 5 \mathrm{~m} \text { thick at base, overlain by } \sim 8 \\
\mathrm{~m} \text { well-sorted, fine reddish sand fining upward to sandy silt. } \\
\text { Three samples from interval 5-7 } \mathrm{m} \text { above river }\end{array}$ & $\begin{array}{l}\text { C } \\
\text { B } \\
\text { A }\end{array}$ & $\mathrm{X}$ \\
\hline 01BL-42 & $\begin{array}{l}\text { W-central Pegram quad, S } \\
\text { side Bear River; same } \\
\text { locality as 99BL-58 but } \\
\text { lower in section }\end{array}$ & $42^{\circ} 12.031^{\prime}$ & $111^{\circ} 14.491^{\prime}$ & $\begin{array}{l}\sim 1865 \\
\text { from map }\end{array}$ & $\begin{array}{l}\text { 35-m section in Bear River and side-fan fluvial deposits and lake } \\
\text { deposits. Two tephra layers (A, B) in basal alluvium. Marl bed } \\
\text { (C) in center of section, overlain by cemented fan-delta and } \\
\text { lacustrine mud; overlain by loess. Units are meters above base of } \\
\text { section }\end{array}$ & $\begin{array}{l}\text { 99BL58B } \\
\text { 99BL58A } \\
\text { C, } 11.8 \mathrm{~m} \\
\text { B, } 1.0 \mathrm{~m} \\
\text { A, } 0.0 \mathrm{~m}\end{array}$ & $\begin{array}{l}X \\
X \\
X\end{array}$ \\
\hline $\begin{array}{l}\text { BL00-02 } \\
\text { (DK99-18) }\end{array}$ & $\begin{array}{l}\text { North Eden canyon, W of } \\
\text { highway, NE Bear Lake } \\
\text { South quad }\end{array}$ & $41^{\circ} 59.38^{\prime}$ & $111^{\circ} 15.95^{\prime}$ & $\begin{array}{l}1814 \\
\text { from map }\end{array}$ & $\begin{array}{l}\text { 0-50: Red, massive, unsorted, imbricated alluvium } \\
\text { 50-190: Pink, massive marl with Stagnicola shells } \\
\text { 190-210: Gray, bedded, sorted, rounded, lacustrine gravel } \\
\text { 210-250: Gray, massive marl with Stagnicola shells }\end{array}$ & DK99-18A & \\
\hline BL00-02C & $\begin{array}{l}\text { North Eden canyon, } \mathrm{N} \text { of } \\
\text { creek, NE Bear Lake South } \\
\text { quad }\end{array}$ & $41^{\circ} 59.38^{\prime}$ & $111^{\circ} 15.95^{\prime}$ & $\begin{array}{l}1830 \\
\text { from map }\end{array}$ & $\begin{array}{l}\text { 0-40: Pink and green massive marl } \\
\text { 40-100: Greenish-gray, massive marl with Valvata and Stagnicola } \\
\text { shells } \\
\text { 100-250: Red, massive, unsorted, angular colluvium }\end{array}$ & DK98-03A, B & \\
\hline
\end{tabular}


Table 1. Description of measured sections, auger holes, and sediment samples from the Bear Lake drainage basin, 1998-2001-Continued.

\begin{tabular}{|c|c|c|c|c|c|c|c|}
\hline $\begin{array}{l}\text { Sample } \\
\text { Number }^{1}\end{array}$ & General location & Latitude & Longitude & $\begin{array}{l}\text { Surface } \\
\text { altitude } \\
\text { (m) }\end{array}$ & $\begin{array}{l}\text { Description of unit } \\
\text { (depth below top of section, in cm except as noted) }\end{array}$ & $\begin{array}{l}\text { Sample } \\
\text { depth }(\mathrm{cm})\end{array}$ & $\begin{array}{l}\text { Split for } \\
\text { ostracodes? } \\
\text { (see table 3) }\end{array}$ \\
\hline BL00-07 & $\begin{array}{l}\text { Bee Hunt hollow, E of } \\
\text { highway, NE Bear Lake } \\
\text { North quad }\end{array}$ & $42^{\circ} 04.07^{\prime}$ & $111^{\circ} 15.10^{\prime}$ & $\begin{array}{l}1830 \\
\text { from map }\end{array}$ & $\begin{array}{l}\text { 0-160: Reddish-brown, massive lacustrine mud } \\
\text { 160-180: White, massive marl with Stagnicola shells } \\
\text { 180-230: Grayish-green, massive, lacustrine mud with Stagnicola } \\
\text { and Valvata shells } \\
\text { 230-265: Greenish-brown, massive lacustrine mud } \\
\text { 265-310: Red, massive, lacustrine mud }\end{array}$ & DK99-20C & \\
\hline $\begin{array}{l}\text { BL00-08 } \\
(00 B L-37)\end{array}$ & $\begin{array}{l}\text { Bedrock gully, E of Mud } \\
\text { Lake, SE Dingle quad }\end{array}$ & $42^{\circ} 08.849^{\prime}$ & $111^{\circ} 15.723^{\prime}$ & 1845 & $\begin{array}{l}\text { 0-80: Brown, massive, unsorted colluvium } \\
\text { 80-150: Gray, bedded, sorted, angular lacustrine gravel } \\
\text { 150-300: Brown, massive, unsorted colluvium }\end{array}$ & & \\
\hline $\begin{array}{l}\text { BL00-09 } \\
(99 \mathrm{BL}-25)\end{array}$ & $\begin{array}{l}\text { Rainbow canal, W of Dingle, } \\
\text { NE Dingle quad }\end{array}$ & $42^{\circ} 13.14^{\prime}$ & $111^{\circ} 17.75^{\prime}$ & 1810.372 & $\begin{array}{l}\text { 0-70: Dark gray, weakly bedded, organic-rich; Lymnaea shells } \\
\text { 70-110: Pink, massive silty marl } \\
\text { 110-140: Sorted fine to medium sand, gradual upper contact } \\
\text { 140-250: Pink, bedded, sorted, sandy gravel }\end{array}$ & $\begin{array}{l}60-70 \mathrm{~cm} \\
\text { DK98-02A }\end{array}$ & \\
\hline BL00-10 & $\begin{array}{l}\text { Cisco Beach, exposure above } \\
\text { beach, NE Bear Lake South } \\
\text { quad }\end{array}$ & $41^{\circ} 58.45^{\prime}$ & $111^{\circ} 16.17^{\prime}$ & $\begin{array}{l}1814 \\
\text { from map }\end{array}$ & $\begin{array}{l}\text { 0-180: Gray, bedded, sorted, rounded, beach gravel with } \\
\text { Stagnicola and Carinifex shells } \\
\text { 180-215: Pink, massive, lacustrine mud } \\
\text { 215-320: Gray, weakly bedded, marl }\end{array}$ & & \\
\hline BL00-11 & $\begin{array}{l}\text { Hen House, E of highway, } \\
\text { NE Bear Lake N quad }\end{array}$ & $42^{\circ} 05.02^{\prime}$ & $111^{\circ} 15.28^{\prime}$ & $\begin{array}{l}1830 \\
\text { from map }\end{array}$ & $\begin{array}{l}\text { 0-60: Brown, massive, silty colluvium } \\
\text { 60-300: White and gray, massive, marl with Stagnicola shells }\end{array}$ & BL00-11 & \\
\hline BL00-12 & $\begin{array}{l}\text { Bear Hollow pit, E of US 89, } \\
\text { SE Montpelier quad }\end{array}$ & $42^{\circ} 16.27^{\prime}$ & $111^{\circ} 16.98^{\prime}$ & $\begin{array}{l}1832 \\
\text { from map }\end{array}$ & $\begin{array}{l}\text { 0-20: Red, massive loess } \\
\text { 20-110: Brown, massive, unsorted, imbricated, coarse-grained } \\
\text { alluvial sand and gravel; 20-cm-thick Bk horizon (stage I) } \\
\text { 110-165: Brown, massive, organic-rich mud } \\
\text { 165-200: Brown, massive, unsorted, imbricated, coarse alluvial } \\
\text { sand and gravel; 30-cm-thick Bk horizon (stage II) } \\
\text { 200-350: Covered } \\
\text { 350-380: Brown, massive, unsorted, imbricated, coarse alluvial } \\
\text { sand and gravel; 30-cm-thick Bk horizon (stage I) } \\
\text { 380-395: Yellow and brown, bedded, gently dipping, fan-delta } \\
\text { coarse sand with Stagnicola and Valvata shells } \\
\text { 395-435: Brown, massive, unsorted, imbricated, coarse alluvial } \\
\text { sand and gravel; 20-cm-thick Bk horizon (stage II) } \\
\text { 435-460: Gray and brown, bedded, gently dipping, fan-delta } \\
\text { coarse sand and gravel with Stagnicola and Valvata shells } \\
\text { 460-500: Brown, massive, unsorted, imbricated, coarse alluvium; } \\
\text { 35-cm-thick Bk horizon (stage II+) }\end{array}$ & & \\
\hline
\end{tabular}


Table 1. Description of measured sections, auger holes, and sediment samples from the Bear Lake drainage basin, 1998-2001-Continued.

\begin{tabular}{|c|c|c|c|c|c|c|c|}
\hline $\begin{array}{l}\text { Sample } \\
\text { Number }^{1}\end{array}$ & General location & Latitude & Longitude & $\begin{array}{l}\text { Surface } \\
\text { altitude } \\
(\mathrm{m})\end{array}$ & $\begin{array}{l}\text { Description of unit } \\
\text { (depth below top of section, in cm except as noted) }\end{array}$ & $\begin{array}{l}\text { Sample } \\
\text { depth }(\mathrm{cm})\end{array}$ & $\begin{array}{l}\text { Split for } \\
\text { ostracodes? } \\
\text { (see table 3) }\end{array}$ \\
\hline BL00-13 & $\begin{array}{l}\text { Bennington, } \mathrm{N} \text { of Pescadero, } \\
\text { SW Georgetown quad }\end{array}$ & $42^{\circ} 23.80^{\prime}$ & $111^{\circ} 21.20^{\prime}$ & $\begin{array}{l}1823 \\
\text { from map }\end{array}$ & $\begin{array}{l}\text { 0-100: Yellow, massive, unsorted, imbricated, coarse alluvium } \\
\text { 100-115: Greenish-brown, massive, lacustrine mud } \\
\text { 115-125: White, massive marl } \\
\text { 125-185: Yellowish-green, massive, lacustrine silt } \\
\text { 185-200: White, massive marl } \\
\text { 200-260: Red, massive marl } \\
\text { 260-330: Brown, weakly bedded, lacustrine, silty sand } \\
\text { 330-350: Brown, massive, lacustrine silt; Sphaerium shells }\end{array}$ & DK97-10A & $\begin{array}{l}X \\
X\end{array}$ \\
\hline $\begin{array}{l}\text { BL00-14 } \\
(99 \mathrm{BL}-84) \\
\text { not on } \\
\text { Figure } 1\end{array}$ & $\begin{array}{l}\text { Georgetown gravel pit, W of } \\
\text { Georgetown, NE Nounan } \\
\text { quad }\end{array}$ & $42^{\circ} 28.83^{\prime}$ & $111^{\circ} 24.00^{\prime}$ & $\begin{array}{l}1826 \\
\text { from map }\end{array}$ & $\begin{array}{l}\text { 0-200: Gray, massive marl with Stagnicola shells } \\
\text { 200-285: Yellow, weakly bedded, fine- to medium-grained } \\
\text { lacustrine sand } \\
\text { 285-305: Gray, massive, lacustrine mud } \\
\text { 305-355: Yellow, bedded, rounded, alluvial sand and gravel with } \\
\text { Sphaerium shells } \\
\text { 355-400: Yellow, planar-laminated, coarse alluvial sand } \\
\text { 00-455: Reddish-brown, planar cross-bedded, coarse-grained } \\
\text { alluvial sand } \\
\text { 455-660: Yellow, planar cross-bedded, fluvial sand and rounded } \\
\text { quartzite-rich gravel with Sphaerium shells } \\
\text { 660-700: Yellowish-red, ripple laminated medium-grained fluvial } \\
\text { sand } \\
\text { 700-780: Yellow, planar cross-bedded, fluvial sand and rounded } \\
\text { quartzite-rich gravel }\end{array}$ & DK99-28C & $X$ \\
\hline $\begin{array}{l}\text { BL00-15 } \\
\text { Not on } \\
\text { Figure } 1\end{array}$ & $\begin{array}{l}\text { Nounan Road, divide } \\
\text { between Bear Lake and } \\
\text { Nounan valleys, NE } \\
\text { Nounan quad }\end{array}$ & $42^{\circ} 27.00^{\prime}$ & $111^{\circ} 23.75^{\prime}$ & $\begin{array}{l}1828 \\
\text { from map }\end{array}$ & $\begin{array}{l}\text { 0-90: Red, massive loess } \\
\text { 90-225: Yellowish-brown, weakly cross-bedded, medium-grained } \\
\text { beach sand with Sphaerium shells } \\
\text { 225-300: Gray, massive marl }\end{array}$ & & \\
\hline $\begin{array}{l}\text { BL00-17 } \\
\text { Not on } \\
\text { Figure } 1\end{array}$ & $\begin{array}{l}\text { Highway } 30 \text { cut } \mathrm{N} \text { of Bear } \\
\text { Lake-Caribou County line, } \\
\text { E of highway, NW Fossil } \\
\text { Canyon quad }\end{array}$ & $42^{\circ} 35.42^{\prime}$ & $111^{\circ} 29.33^{\prime}$ & $\begin{array}{l}1828 \\
\text { from map }\end{array}$ & $\begin{array}{l}\text { 0-90: Red, massive loess. } \\
\text { 90-355: Yellow, massive, slightly weathered marl with Stagnicola } \\
\text { shells } \\
\text { 355-465: Yellow, massive, sorted, rounded, imbricated, alluvial } \\
\text { sand and gravel. }\end{array}$ & & $X$ \\
\hline $\begin{array}{l}\text { BL00-18 } \\
\text { Not on } \\
\text { Figure } 1\end{array}$ & $\begin{array}{l}\text { Highway } 30 \text { cut } \mathrm{S} \text { of Bear } \\
\text { Lake-Caribou County line, } \\
\text { E of highway, NW Fossil } \\
\text { Canyon quad }\end{array}$ & $42^{\circ} 35.25^{\prime}$ & $111^{\circ} 29.00^{\prime}$ & $\begin{array}{l}1828 \\
\text { from map }\end{array}$ & $\begin{array}{l}\text { 0-165: Yellow, massive marl; Stagnicola and Valvata shells } \\
\text { 165-240: Yellow, massive, angular, alluvial sand and gravel } \\
\text { 240-250: Gray, massive marl } \\
\text { 250-275: Yellow, planar cross-bedded, sorted, medium-grained } \\
\text { alluvial sand }\end{array}$ & & $X$ \\
\hline
\end{tabular}


Table 1. Description of measured sections, auger holes, and sediment samples from the Bear Lake drainage basin, 1998-2001-Continued.

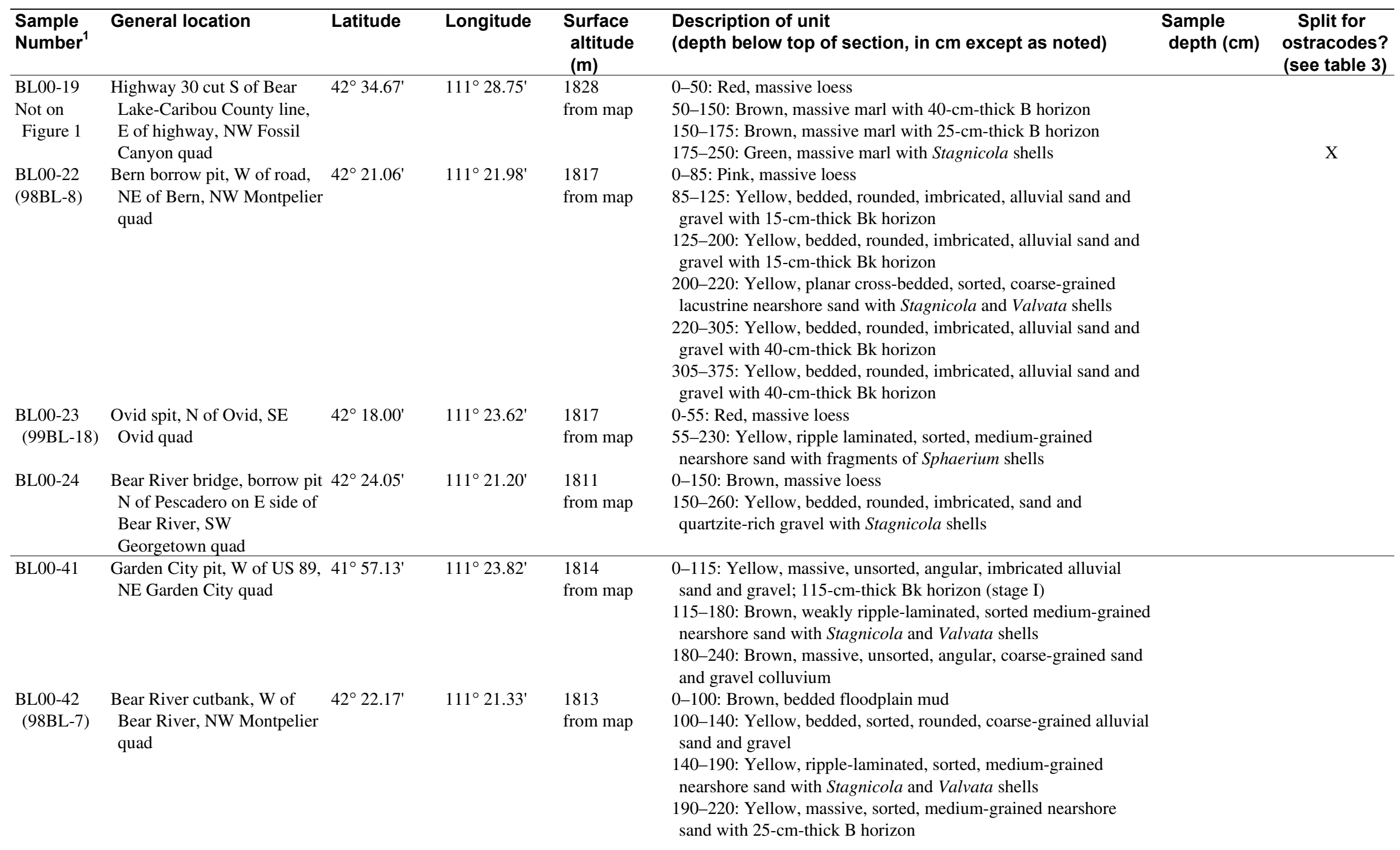


Table 1. Description of measured sections, auger holes, and sediment samples from the Bear Lake drainage basin, 1998-2001—Continued.

\begin{tabular}{|c|c|c|c|c|c|c|c|}
\hline $\begin{array}{l}\text { Sample } \\
\text { Number }\end{array}$ & General location & Latitude & Longitude & $\begin{array}{l}\text { Surface } \\
\text { altitude } \\
\text { (m) }\end{array}$ & $\begin{array}{l}\text { Description of unit } \\
\text { (depth below top of section, in } \mathrm{cm} \text { except as noted) }\end{array}$ & $\begin{array}{l}\text { Sample } \\
\text { depth }(\mathrm{cm})\end{array}$ & $\begin{array}{c}\text { Split for } \\
\text { ostracodes? } \\
\text { (see table 3) }\end{array}$ \\
\hline DK93-23 & $\begin{array}{l}\text { Mud Lake just north of dam; } \\
\text { sampled as outcrop when } \\
\text { control structure broke in } \\
1993\end{array}$ & $\begin{array}{l}42^{\circ} 07.4^{\prime} \\
\text { from map }\end{array}$ & $\begin{array}{l}111^{\circ} 18.8^{\prime} \\
\text { from map }\end{array}$ & $\begin{array}{l}1804 \\
\text { from map }\end{array}$ & $\begin{array}{l}\text { 0-100: Peat and organic-rich silt with shell hash layers } \\
\text { 100-170: Dark gray fine and medium sand; layers of snails } \\
\text { 170-190: Organic-rich silt and peat with silty sand layers; snail } \\
\text { shells and shell hash } \\
\text { 190-270: Laminated marl, rhythmically bedded gray silt and white } \\
\text { silt-clay; abundant snails } \\
\text { 270-280: Sedge peat }\end{array}$ & & $\begin{array}{l}X \\
X\end{array}$ \\
\hline DK96-06 & $\begin{array}{l}\text { Fish ladder west of Lifton } \\
\text { pumping station, NW Bear } \\
\text { Lake North quad }\end{array}$ & $42^{\circ} 07.35^{\prime}$ & $111^{\circ} 20.15^{\prime}$ & $\begin{array}{l}1806 \\
\text { from map }\end{array}$ & $\begin{array}{l}\text { 0-140: Stratified medium sand with pebble gravel; paleosol (Bw } \\
\text { horizon) at } 30 \mathrm{~cm} \text {; abundant reworked mollusc shells, mainly } \\
\text { Carinifex and Sphaerium; basal contact abrupt } \\
\text { 140-235: Bedded marl and organic-rich mud; abundant in-situ } \\
\text { snails, dominantly Stagnicola and Valvata }\end{array}$ & $\mathrm{X}$ & $X$ \\
\hline
\end{tabular}

'Sample sites beginning with numbers followed by BL, for example 00BL-64, described and sampled by Reheis; first two numbers designate year of collection. Sites beginning with letters described and sampled by Laabs (BL) and Kaufman (DK); middle numbers designate year of collection. Numbers following dashes are site numbers. 
Table 2. Radiocarbon dates from outcrop and auger holes in the Bear Lake drainage basin, 1998-2001.

[cm: centimeters; $\mathbf{m}$ : meters; $\mathbf{y r}:$ year]

\begin{tabular}{|c|c|c|c|c|c|c|c|}
\hline $\begin{array}{l}\text { Sample } \\
\text { Number }\end{array}$ & General location & Latitude & Longitude & Material dated & $\begin{array}{l}\text { Sample } \\
\text { depth } \\
(\mathrm{cm})\end{array}$ & $\begin{array}{l}{ }^{14} \mathrm{C} \text { lab } \\
\text { number }\end{array}$ & $\begin{array}{l}\text { Radiocarbon } \\
\text { age (yr) }\end{array}$ \\
\hline 99BL-26B & Rainbow Canal $\mathrm{N}$ of airport road bridge & $42^{\circ} 13.992^{\prime}$ & $111^{\circ} 49.166^{\prime}$ & Gastropod shells & $80-100$ & WW-3049 & $1,400 \pm 40$ \\
\hline 99BL-34 & Auger hole, $\mathrm{S}$ side of road east of Bloomington & $42^{\circ} 11.0637^{\prime}$ & $111^{\circ} 22.6253^{\prime}$ & Gastropod shells & $0-50$ & WW-2583 & $7,760 \pm 70$ \\
\hline \multirow[t]{3}{*}{ 99BL-37 } & \multirow{3}{*}{$\begin{array}{l}\text { Auger hole, E of Bloomington, up side of fault } \\
\text { cutting meander }\end{array}$} & \multirow[t]{3}{*}{$42^{\circ} 12.7752^{\prime}$} & \multirow[t]{3}{*}{$111^{\circ} 21.8142^{\prime}$} & Shell fragments & $70-80$ & WW-2584 & $1,955 \pm 70$ \\
\hline & & & & Gastropod shells & $80-100$ & WW-2585 & $10,420 \pm 80$ \\
\hline & & & & Gastropod shells & $200-230$ & WW-2586 & $9,820 \pm 75$ \\
\hline \multirow[t]{2}{*}{ 99BL-38 } & \multirow{2}{*}{$\begin{array}{l}\text { Auger hole on Paris-Dingle road, W of outlet } \\
\text { canal, Bear R. abandoned channel 2? }\end{array}$} & \multirow[t]{2}{*}{$42^{\circ} 14.1401^{\prime}$} & \multirow[t]{2}{*}{$111^{\circ} 21.6109^{\prime}$} & Gastropod shells & $0-20$ & WW-2587 & $2,645 \pm 55$ \\
\hline & & & & Gastropod shells & $230-250$ & WW-2588 & $7,985 \pm 70$ \\
\hline 99BL-39 & $\begin{array}{l}\text { Auger hole E of airport, Bear River abandoned } \\
\text { channel 3? }\end{array}$ & $42^{\circ} 15.1191^{\prime}$ & $111^{\circ} 19.4917^{\prime}$ & Gastropod shells & $60-70$ & WW-2589 & $2,445 \pm 55$ \\
\hline \multirow[t]{2}{*}{ 99BL-42 } & \multirow{2}{*}{$\begin{array}{l}\text { Auger hole, W of outlet, } \mathrm{N} \text { of Mud Lake dam; } \\
\text { Bear River or Bear Lake channel? }\end{array}$} & \multirow[t]{2}{*}{$42^{\circ} 12.8604^{\prime}$} & \multirow[t]{2}{*}{$111^{\circ} 20.5828^{\prime}$} & Gastropod shells & $0-25$ & WW-590 & $1,720 \pm 55$ \\
\hline & & & & Gastropod shells & $200-220$ & WW-2591 & $12,220 \pm 100$ \\
\hline \multirow[t]{3}{*}{ 99BL-45 } & \multirow{3}{*}{$\begin{array}{l}\text { Cut, W side Rainbow Canal. Bear River } \\
\text { abandoned channel } 3\end{array}$} & \multirow[t]{3}{*}{$42^{\circ} 14.2702^{\prime}$} & \multirow[t]{3}{*}{$111^{\circ} 17.6390^{\prime}$} & Gastropod shells & $60-65$ & WW-2592 & $5,130 \pm 65$ \\
\hline & & & & Gastropod shells & $120-130$ & WW-2593 & $8,520 \pm 70$ \\
\hline & & & & Bivalve shells & $200-220$ & WW-2594 & $11,015 \pm 85$ \\
\hline 99BL-47 & Cut, Rainbow Canal, S of airport road & $42^{\circ} 13.0010^{\prime}$ & $111^{\circ} 17.7616^{\prime}$ & Gastropod shells & $0-80$ & WW-2595 & $7,150 \pm 70$ \\
\hline 99BL-48A & Cut, Rainbow Canal, S of airport road & $42^{\circ} 12.6575^{\prime}$ & $111^{\circ} 17.048^{\prime}$ & Gastropod shells & $165-185$ & WW-3048 & $8,460 \pm 40$ \\
\hline 99BL-48B & Cut, Rainbow Canal, S of airport road & $42^{\circ} 12.6540^{\prime}$ & $111^{\circ} 17.7060^{\prime}$ & Gastropod shells & $110-140$ & WW-3050 & $7,870 \pm 40$ \\
\hline \multirow[t]{2}{*}{ 99BL-49 } & \multirow{2}{*}{$\begin{array}{l}\text { Auger hole, Rainbow Canal. Bear River } \\
\text { abandoned channel } 1\end{array}$} & \multirow[t]{2}{*}{$42^{\circ} 12.4719^{\prime}$} & \multirow[t]{2}{*}{$111^{\circ} 17.7213^{\prime}$} & \multirow[t]{2}{*}{ Gastropod shells } & $20-50$ & WW-2596 & $4,000 \pm 60$ \\
\hline & & & & & $270-300$ & WW-2597 & $6,925 \pm 70$ \\
\hline \multirow[t]{2}{*}{ 99BL-59 } & \multirow{2}{*}{$\begin{array}{l}\text { Drainage-ditch cut, along fault S of Dingle, E- } \\
\text { central Dingle quad }\end{array}$} & \multirow[t]{2}{*}{$42^{\circ} 11.6398^{\prime}$} & \multirow[t]{2}{*}{$111^{\circ} 16.0818^{\prime}$} & \multirow[t]{2}{*}{ Shell fragments } & $\mathrm{B}, 180$ & WW-2599 & $40,800 \pm 1,600$ \\
\hline & & & & & A, $200-250$ & WW-2598 & $\begin{array}{l}39,100 \pm 1,100 \\
\text { (minimum age) }\end{array}$ \\
\hline 00BL-27 & $\begin{array}{l}\text { Outlet canal S of Hwy 89, W-central Montpelier } \\
\text { quad }\end{array}$ & $\begin{array}{l}\sim 42^{\circ} 30.0^{\prime} \\
\text { from map }\end{array}$ & $\begin{array}{l}\sim 111^{\circ} 21.5^{\prime} \\
\text { from map }\end{array}$ & Gastropod shells & $10-20$ & WW-3047 & $4,880 \pm 40$ \\
\hline 00BL-63A & $\begin{array}{l}\text { Waterline trench } \mathrm{E} \text { of road, } \mathrm{S} \text { of Indian Creek, } \\
\text { NE Bear Lake N quad }\end{array}$ & $42^{\circ} 05.1811^{\prime}$ & $111^{\circ} 15.3086^{\prime}$ & $\begin{array}{l}\text { Gastropod and bivalve } \\
\text { shells }\end{array}$ & Several meters & WW-3369 & $39,870 \pm 490$ \\
\hline 00BL-64 & $\begin{array}{l}\text { Borrow pit S of Indian Creek, NE Bear Lake N } \\
\text { quad }\end{array}$ & $\begin{array}{l}\sim 42^{\circ} 05.3 \\
\text { from map }\end{array}$ & $\begin{array}{l}\sim 111^{\circ} 15.4 \\
\text { from map }\end{array}$ & $\begin{array}{l}\text { Gastropod and bivalve } \\
\text { shells }\end{array}$ & $100-120$ & WW-3370 & $10,810 \pm 40$ \\
\hline 01BL-34 & $\begin{array}{l}\text { Borrow pit W of US-30, Bear Hollow, SE } \\
\text { Montpelier quad }\end{array}$ & $\sim 42^{\circ} 16.5^{\prime}$ & $\sim 111^{\circ} 17^{\prime}$ & Gastropod shells & 350 & WW-3721 & $45,950 \pm 1,020$ \\
\hline 01BL-35 & $\begin{array}{l}\text { Bear River cutbank N of US 30, W-central } \\
\text { Montpelier quad }\end{array}$ & $42^{\circ} 19.81^{\prime}$ & $111^{\circ} 23.26^{\prime}$ & Gastropod shells & $\begin{array}{l}\text { B, } 150-190 \\
\text { A, } 190-250\end{array}$ & $\begin{array}{l}\text { WW-3723 } \\
\text { WW-3722 }\end{array}$ & $\begin{array}{l}13,675 \pm 50 \\
16,350 \pm 50\end{array}$ \\
\hline DK93-23D & $\begin{array}{l}\text { Mud Lake north of Lifton dam control structure, } \\
\text { N-central Bear Lake N quad }\end{array}$ & $\begin{array}{l}42^{\circ} 07.4^{\prime} \\
\text { from map }\end{array}$ & $\begin{array}{l}111^{\circ} 18.8^{\prime} \\
\text { from map }\end{array}$ & Carinifex shell & 130 & NSRL-10572 & $6,750 \pm 60$ \\
\hline DK96-01 & $\begin{array}{l}\text { Cisco Beach, exposure into an 8-m-high terrace, } \\
\text { NE Bear Lake South quad }\end{array}$ & $41^{\circ} 58.45^{\prime}$ & $111^{\circ} 16.17^{\prime}$ & Mollusk shell & 150 & WW-1557 & $10,420 \pm 50$ \\
\hline DK96-06D & $\begin{array}{l}\text { Fish ladder, W of Lifton pumping station, NW } \\
\text { Bear Lake North quad }\end{array}$ & $42^{\circ} 07.35^{\prime}$ & $111^{\circ} 20.15^{\prime}$ & Stagnicola shell & 70 & NSRL-1566 & $7,210 \pm 40$ \\
\hline
\end{tabular}


Table 2. Radiocarbon dates from outcrop and auger holes in the Bear Lake drainage basin, 1998-2001—Continued.

\begin{tabular}{|c|c|c|c|c|c|c|c|}
\hline $\begin{array}{l}\text { Sample } \\
\text { Number }\end{array}$ & General location & Latitude & Longitude & Material dated & $\begin{array}{l}\text { Sample } \\
\text { depth } \\
\text { (cm) }\end{array}$ & $\begin{array}{l}{ }^{14} \mathrm{C} \text { lab } \\
\text { number }\end{array}$ & $\begin{array}{l}\text { Radiocarbon } \\
\text { age (yr) }\end{array}$ \\
\hline DK96-06B & $\begin{array}{l}\text { Fish ladder, W of Lifton pumping station, NW } \\
\text { Bear Lake North quad }\end{array}$ & $42^{\circ} 07.35^{\prime}$ & $111^{\circ} 20.15^{\prime}$ & Stagnicola shell & $150-160$ & WW-1561 & $5,650 \pm 40$ \\
\hline DK96-06B & $\begin{array}{l}\text { Fish ladder, W of Lifton pumping station, NW } \\
\text { Bear Lake North quad }\end{array}$ & $42^{\circ} 07.35^{\prime}$ & $111^{\circ} 20.15^{\prime}$ & Stagnicola shell & $150-160$ & NSRL-1566 & $5,580 \pm 50$ \\
\hline DK96-06B & $\begin{array}{l}\text { Fish ladder, W of Lifton pumping station, NW } \\
\text { Bear Lake North quad }\end{array}$ & $42^{\circ} 07.35^{\prime}$ & $111^{\circ} 20.15^{\prime}$ & Charcoal & $150-160$ & WW-1566 & $5,530 \pm 50$ \\
\hline DK96-06A & $\begin{array}{l}\text { Fish ladder, W of Lifton pumping station, NW } \\
\text { Bear Lake North quad }\end{array}$ & $42^{\circ} 07.35^{\prime}$ & $111^{\circ} 20.15^{\prime}$ & Valvata shell & $210-220$ & NSRL-10940 & $8,520 \pm 65$ \\
\hline DK96-06A & $\begin{array}{l}\text { Fish ladder, W of Lifton pumping station, NW } \\
\text { Bear Lake North quad }\end{array}$ & $42^{\circ} 07.35^{\prime}$ & $111^{\circ} 20.15^{\prime}$ & Valvata shell & $210-220$ & NSRL-10941 & $8,550 \pm 65$ \\
\hline DK97-10A & $\begin{array}{l}\text { Bennington, } \mathrm{N} \text { of Pescadero, SW Georgetown } \\
\text { quad }\end{array}$ & $42^{\circ} 23.80^{\prime}$ & $111^{\circ} 21.20^{\prime}$ & Sphaerium shell & Several meters & WW-1559 & $>43,260$ \\
\hline DK98-02A & Rainbow canal, W of Dingle, NE Dingle quad & $42^{\circ} 14.27^{\prime}$ & $111^{\circ} 17.63^{\prime}$ & Stagnicola shell & $200-250$ & NSRL-10569 & $8,350 \pm 70$ \\
\hline DK98-03A & $\begin{array}{l}\text { Road cut north of North Eden canyon, NE Bear } \\
\text { Lake south quad }\end{array}$ & $41^{\circ} 59.53^{\prime}$ & $111^{\circ} 15.88^{\prime}$ & Stagnicola; 2 shells & several meters & NSRL-10570 & $37,900 \pm 460$ \\
\hline DK98-03B & $\begin{array}{l}\text { Road cut north of North Eden canyon, NE Bear } \\
\text { Lake south quad }\end{array}$ & $41^{\circ} 59.53^{\prime}$ & $111^{\circ} 15.88^{\prime}$ & Stagnicola shell & several meters & NSRL-10571 & $38,700 \pm 790$ \\
\hline DK99-11 & Ovid spit, $\mathrm{N}$ of Ovid, SE Ovid quad & $42^{\circ} 18.00^{\prime}$ & $111^{\circ} 23.62^{\prime}$ & Shell fragments & $180-210$ & NSRL-11353 & $41,240 \pm 640$ \\
\hline DK99-13 & $\begin{array}{l}\text { Bear River cutbank, W of Bear River, NW } \\
\text { Montpelier quad }\end{array}$ & $42^{\circ} 22.17^{\prime}$ & $111^{\circ} 21.33^{\prime}$ & Stagnicola shell & $170-180$ & NSRL-11354 & $36,800 \pm 790$ \\
\hline DK99-18A & $\begin{array}{l}\text { North Eden canyon, W of highway, NE Bear } \\
\text { Lake south quad }\end{array}$ & $41^{\circ} 59.22^{\prime}$ & $111^{\circ} 15.56^{\prime}$ & Charcoal & $50-150$ & NSRL-11355 & $8,780 \pm 90$ \\
\hline DK99-18B & $\begin{array}{l}\text { North Eden canyon, W of highway, NE Bear } \\
\text { Lake south quad }\end{array}$ & $41^{\circ} 59.38^{\prime}$ & $111^{\circ} 15.95^{\prime}$ & Discus shell & $50-150$ & NSRL-11356 & $10,490 \pm 100$ \\
\hline DK99-19B & $\begin{array}{l}\text { North Eden canyon, W of highway, NE Bear } \\
\text { Lake south quad }\end{array}$ & $41^{\circ} 59.38^{\prime}$ & $111^{\circ} 15.95^{\prime}$ & Discus shells & $50-160(?)$ & NSRL-11357 & $10640 \pm 80$ \\
\hline DK99-20C & $\begin{array}{l}\text { Bee Hunt hollow, E of highway, NE Bear Lake } \\
\text { North quad }\end{array}$ & $42^{\circ} 04.07^{\prime}$ & $111^{\circ} 15.10^{\prime}$ & 3 Stagnicola shells & $80-130$ & NSRL-11358 & $44,240 \pm 730$ \\
\hline DK99-28C & $\begin{array}{l}\text { Georgetown gravel pit (site BL00-14), W of } \\
\text { Georgetown, NE Nounan quad }\end{array}$ & $42^{\circ} 28.83^{\prime}$ & $111^{\circ} 24.00^{\prime}$ & Discus shell & $100-140$ & NSRL-11359 & $>45,200$ \\
\hline BL-00-11 & $\begin{array}{l}\text { Hen House, E of highway, NE Bear Lake North } \\
\text { quad }\end{array}$ & $42^{\circ} 05.02^{\prime}$ & $111^{\circ} 15.28^{\prime}$ & Sphaerium shell & $100-150$ & NSRL-12061 & $36,000 \pm 320$ \\
\hline BL-00-41A & $\begin{array}{l}\text { Garden City pit, W of US 89, NE Garden City } \\
\text { quad }\end{array}$ & $41^{\circ} 57.13^{\prime}$ & $111^{\circ} 23.82^{\prime}$ & Mollusk shell & $125-160$ & NSRL-12062 & $13,540 \pm 70$ \\
\hline BL-00-41B & $\begin{array}{l}\text { Garden City pit, W of US 89, NE Garden City } \\
\text { quad }\end{array}$ & $41^{\circ} 57.13^{\prime}$ & $111^{\circ} 23.82^{\prime}$ & Mollusk shell & $125-160$ & NSRL-12063 & $13,280 \pm 70$ \\
\hline BL-00-42 & $\begin{array}{l}\text { Bear River cutbank, W of Bear River, NW } \\
\text { Montpelier quad }\end{array}$ & $42^{\circ} 22.17^{\prime}$ & $111^{\circ} 21.33^{\prime}$ & Stagnicola shells & $175-185$ & NSRL-12064 & $44,300 \pm 920$ \\
\hline
\end{tabular}

${ }^{1}$ Letter prefixes indicate laboratory: WW-, U.S. Geological Survey, Reston, Va.; NSRL-, Institute of Arctic and Alpine Research, Boulder, Colo. 
Table 3. Ostracodes in sediment samples from outcrops in the Bear Lake drainage basin, 1998-2001.

[cm: centimeters; m: meters]

\begin{tabular}{|c|c|c|c|c|c|c|}
\hline $\begin{array}{l}\text { Site and } \\
\text { sample } \\
\text { number }\end{array}$ & $\begin{array}{l}\text { Description of unit } \\
\text { (see table 1) }\end{array}$ & $\begin{array}{l}\text { Depth below } \\
\text { top of } \\
\text { section (cm } \\
\text { unless } \\
\text { noted) }\end{array}$ & $\begin{array}{l}\text { Age } \\
\text { (if dated; } \\
\text { see table } \\
\text { 2) }\end{array}$ & Ostracode taxa & Other taxa & Comments \\
\hline $98 \mathrm{BL}-5$ & $\begin{array}{l}\text { Base of paleosol formed in } \\
\text { marl }\end{array}$ & $192-215$ & & None & & \\
\hline 99BL-35 & $\begin{array}{l}\text { Massive silt and sand; } \\
\text { bedded fine sand at base }\end{array}$ & $190-210$ & & None & & \\
\hline 99BL-35 & $\begin{array}{l}\text { Oxidized, bedded calcareous } \\
\text { marl }\end{array}$ & $390-410$ & & None & & \\
\hline 99BL-35 & $\begin{array}{l}\text { Oxidized, bedded calcareous } \\
\text { marl; interbedded sand } \\
\text { laminae }\end{array}$ & $430-450$ & & None & & \\
\hline 99BL-37 & $\begin{array}{l}\text { Calcareous silt and fine sand, } \\
\text { orange mottled, shelly; } \\
\text { overlies reddish, well-sorted } \\
\text { fine sand, with shells }\end{array}$ & $80-100$ & $10,420 \pm 80$ & $\begin{array}{l}\text { Ilyocypris bradyi } \\
\text { Limnocythere itasca } \\
\text { Cypridopsis vidua } \\
\text { Candona caudata } \\
\text { Candona sp. aff. C. rawsoni }\end{array}$ & $\begin{array}{l}\text { Mollusk shell } \\
\text { fragments }\end{array}$ & $\begin{array}{l}\text { All of the taxa are juveniles that were probably } \\
\text { reworked from a wetland sediment }\end{array}$ \\
\hline 99BL-42 & $\begin{array}{l}\text { Reddish fine sand, some } \\
\text { shells }\end{array}$ & $140-160$ & & None & $\begin{array}{l}\text { Gastropods, } \\
\text { charophytes }\end{array}$ & Probably a wetland \\
\hline 99BL-43 & $\begin{array}{l}\text { Reddish silt, clay, and fine } \\
\text { sand }\end{array}$ & $155-180$ & & None & & \\
\hline 99BL-43 & $\begin{array}{l}\text { Interbedded? muddy medium } \\
\text { and fine sand, reddish, } \\
\text { slightly to non-calcareous, } \\
\text { monotonous }\end{array}$ & $240-270$ & & $\begin{array}{l}\text { Heterocypris sp. aff. H. } \\
\text { incongruens } \\
\text { Candona sp. aff. C. rawsoni }\end{array}$ & $\begin{array}{l}\text { Mollusk shell } \\
\text { fragments }\end{array}$ & Spring/seep \\
\hline 99BL-45 & $\begin{array}{l}\text { Calcareous, organic-rich silt } \\
\text { and clay, abundant snails } \\
\text { throughout }\end{array}$ & $120-130$ & $8520 \pm 70$ & $\begin{array}{l}\text { Cypridopsis vidua } \\
\text { Candona stagnalis } \\
\text { Limnocythere itasca } \\
\text { Potamocypris sp. } \\
\text { Cyclocypris ovum } \\
\text { Candona } \text { sp.? } \\
\text { Cyclocypris serena }\end{array}$ & $\begin{array}{l}\text { Numerous aquatic } \\
\text { gastropods, } \\
\text { bivalves }\end{array}$ & Wetland \\
\hline 99BL-48A & $\begin{array}{l}\text { Green clay, locally oxidized; } \\
\text { overlain by calcareous pink } \\
\text { marl }\end{array}$ & $165-185$ & $>8460$ & $\begin{array}{l}\text { Candona caudata } \\
\text { Cypris pubera } \\
\text { Cyclocypris serena } \\
\text { Candona distincta } \\
\text { Candona renoensis } \\
\text { Candona stagnalis } \\
\text { Candona sp. } \\
\text { Limnocythere paraornata } \\
\text { Strandesia horridus } \\
\text { Cypria obesa? } \\
\text { Ilyocypris gibba } \\
\text { Candona decora } \\
\text { Dolerocypris sp. } \\
\text { Candona rawsoni } \\
\text { Strandesia } \mathrm{sp} \text {. }\end{array}$ & $\begin{array}{l}\text { Aquatic and } \\
\text { terrestrial } \\
\text { gastropods, } \\
\text { bivalves }\end{array}$ & $\begin{array}{l}\text { Hydrologically complex wetland having springs, } \\
\text { seeps, standing water, and flowing water }\end{array}$ \\
\hline
\end{tabular}


Table 3. Ostracodes in sediment samples from outcrops in the Bear Lake drainage basin, 1998-2001—Continued.

\begin{tabular}{|c|c|c|c|c|c|c|}
\hline $\begin{array}{l}\text { Site and } \\
\text { sample } \\
\text { number }\end{array}$ & $\begin{array}{l}\text { Description of unit } \\
\text { (see table 1) }\end{array}$ & $\begin{array}{l}\text { Depth below } \\
\text { top of } \\
\text { section (cm } \\
\text { unless } \\
\text { noted) }\end{array}$ & $\begin{array}{l}\text { Age } \\
\text { (if dated; } \\
\text { see table } \\
\text { 2) }\end{array}$ & Ostracode taxa & Other taxa & Comments \\
\hline 99BL-49 & $\begin{array}{l}\text { Reddish-gray silt and sand, } \\
\text { abundant plant fragments, slightly } \\
\text { calcareous }\end{array}$ & $190-215$ & $\begin{array}{l}>4000 \\
<6925\end{array}$ & $\begin{array}{l}\text { Ilyocypris bradyi } \\
\text { Limnocythere paraornata } \\
\text { Physocypria globula } \\
\text { Cyclocypris ovum } \\
\text { Cypridopsis vidua } \\
\text { Cavernocypris wardi } \\
\text { Candona sp. } \\
\text { Cyclocypris serena? } \\
\text { Candona stagnalis }\end{array}$ & Gastropods & $\begin{array}{l}\text { Stream and wetland complex with cold flowing } \\
\text { springs }\end{array}$ \\
\hline 99BL-49 & $\begin{array}{l}\text { Gray silty sand, slightly calcareous, } \\
\text { shell fragments }\end{array}$ & $270-300$ & $6925 \pm 70$ & None & & \\
\hline 99BL-53B & $\begin{array}{l}\text { Well-sorted, well-bedded fine- } \\
\text { medium silt and sand }\end{array}$ & $\sim 10 \mathrm{~m}$ & & & & \\
\hline 99BL-53A & Green clay interbeds & $\sim 15 \mathrm{~m}$ & & $\begin{array}{l}\text { Limnocythere paraornata } \\
\text { Candona sp.? }\end{array}$ & & Stream? \\
\hline $\begin{array}{l}\text { 99BL-84 } \\
\text { (same as } \\
\text { BL00-14) }\end{array}$ & Gray, massive marl & $0-200$ & & None & $\begin{array}{l}\text { Root tube } \\
\text { coquina, } \\
\text { gastropods } \\
\text { (Stagnicola) }\end{array}$ & Wetland \\
\hline 00BL-8 & $\begin{array}{l}\text { Mottled orange calcareous mud, thin } \\
\text { fine sand lenses }\end{array}$ & $235-333$ & & None & Gastropods & \\
\hline 00BL-54E & Pale gray, silty lacustrine mudstone & $6.8 \mathrm{~m}$ & & $\begin{array}{l}\text { Cypridopsis vidua } \\
\text { Cyclocypris serena } \\
\text { Candona sp. cf. C. rawsoni } \\
\text { Cytherissa lacustris }\end{array}$ & $\begin{array}{l}\text { Aquatic and } \\
\text { terrestrial } \\
\text { gastropods, } \\
\text { charophytes }\end{array}$ & $\begin{array}{l}\text { Wetland environment with reworked lacustrine } \\
\text { ostracodes, or wetland and lake environments } \\
\text { integrated with sampling }\end{array}$ \\
\hline 00BL-54D & Pale gray, silty lacustrine sand & $9.2 \mathrm{~m}$ & & $\begin{array}{l}\text { Candona } \text { n. sp. } 1 \\
\text { Candona } \text { n. sp. } 2 \\
\text { Candona caudauta }\end{array}$ & Gastropods & $\begin{array}{l}\text { Lacustrine deposition. The two taxa identified } \\
\text { as new species } 1 \text { and } 2 \text { are taxa endemic to } \\
\text { Bear Lake. The presence of Candona caudata } \\
\text { may indicate this site is near the littoral zone } \\
\text { of Bear Lake }\end{array}$ \\
\hline 00BL-54C & Gray lacustrine mudstone & $10.4 \mathrm{~m}$ & & $\begin{array}{l}\text { Pelocypris sp. } \\
\text { Ilyocypris bradyi } \\
\text { Candona sp. cf. C. rawsoni }\end{array}$ & $\begin{array}{l}\text { Aquatic } \\
\text { gastropods, } \\
\text { bivalves, and } \\
\text { fish bone }\end{array}$ & $\begin{array}{l}\text { Stream environment. Pelocyprids are common } \\
\text { stream ostracodes, and ilyocyprids typically } \\
\text { live in flowing water, whether in spring or } \\
\text { stream flow }\end{array}$ \\
\hline 00BL-54B & Brown lacustrine silt and mudstone & $13.2 \mathrm{~m}$ & & None & & \\
\hline 00BL-54A & $\begin{array}{l}\text { Beach gravel interbedded with silty } \\
\text { sand }\end{array}$ & $19.0 \mathrm{~m}$ & & None & & \\
\hline
\end{tabular}


Table 3. Ostracodes in sediment samples from outcrops in the Bear Lake drainage basin, 1998-2001—Continued.

\begin{tabular}{|c|c|c|c|c|c|c|}
\hline $\begin{array}{l}\text { Site and } \\
\text { sample } \\
\text { number }\end{array}$ & $\begin{array}{l}\text { Description of unit } \\
\text { (see table 1) }\end{array}$ & $\begin{array}{l}\text { Depth below } \\
\text { top of } \\
\text { section (cm } \\
\text { unless } \\
\text { noted) }\end{array}$ & $\begin{array}{l}\text { Age } \\
\text { (if dated; } \\
\text { see table } \\
\text { 2) }\end{array}$ & Ostracode taxa & Other taxa & Comments \\
\hline 00BL-59 & $\begin{array}{l}\text { Thin shell-bearing reddish mudstone } \\
\text { capped by fan gravel; overlies } \\
\text { beach gravel }\end{array}$ & & & None & & \\
\hline 00BL-60C & $\begin{array}{l}\text { Reddish mudstone, probably } \\
\text { lacustrine }\end{array}$ & upper & & $\begin{array}{l}\text { Candona sp. cf. C. rawsoni, } \\
\text { juveniles } \\
\text { Candona spp? }\end{array}$ & & Unknown depositional environment \\
\hline 00BL-60B & Gray lacustrine sand & middle & & & & \\
\hline 00BL-60A & Olive-green lacustrine clay & lower & & $\begin{array}{l}\text { Candona sp. cf. C. acutula } \\
\text { ? } \\
\text { Candona spp. ? } \\
\text { Limnocythere spp. ? }\end{array}$ & & $\begin{array}{l}\text { Candonid and limnocytherid species unknown, } \\
\text { some of these taxa resemble Pliocene and } \\
\text { early Pleistocene ostracodes known from large } \\
\text { lakes }\end{array}$ \\
\hline 00BL-61B & $\begin{array}{l}\text { Reddish mudstone, probably } \\
\text { lacustrine }\end{array}$ & upper & & $\begin{array}{l}\text { Reworked candonids, some } \\
\text { species the same as in } \\
\text { 00BL61A }\end{array}$ & & Depositional environment unknown \\
\hline 00BL-61A & Gray lacustrine sand & lower & & $\begin{array}{l}\text { Candona spp? } \\
\text { Limnocythere sappaensis } \\
\text { Limnocythere spp? } \\
\text { Cytherid genus and species } \\
\text { unknown }\end{array}$ & & $\begin{array}{l}\text { Depositional environment unknown, taxa } \\
\text { resemble species known from large Pliocene } \\
\text { lakes in the region }\end{array}$ \\
\hline 00BL-63B & $\begin{array}{l}\text { Gypsiferous lacustrine deposits } \\
\text { grade upward into gypsiferous } \\
\text { spring-discharge deposits }\end{array}$ & upper & & $\begin{array}{l}\text { Cavernocypris wardi } \\
\text { Candona sp.? juveniles }\end{array}$ & $\begin{array}{l}\text { Aquatic and } \\
\text { terrestrial } \\
\text { gastropods }\end{array}$ & Cold flowing spring \\
\hline 00BL-63A & $\begin{array}{l}\text { Gypsiferous lacustrine deposits } \\
\text { interbedded with fan gravel }\end{array}$ & lower & $\begin{array}{c}>39,870+ \\
490\end{array}$ & & & \\
\hline 01BL-23B & Calcareous pink marl & $37-44$ & & Ilyocypris bradyi & $\begin{array}{l}\text { Aquatic and } \\
\text { terrestrial } \\
\text { gastropods, } \\
\text { charophytes }\end{array}$ & Wetland to flowing spring setting \\
\hline 01BL-24C & $\begin{array}{l}\text { Mixed greenish-gray clay and } \\
\text { gravel, unbedded }\end{array}$ & $3.2-4.2 \mathrm{~m}$ & & $\begin{array}{l}\text { Cyclocypris serena } \\
\text { Cyclocypris ovum } \\
\text { Candona sp. cf. C. rawsoni, } \\
\text { juvenile }\end{array}$ & $\begin{array}{l}\text { Terrestrial } \\
\text { gastropods }\end{array}$ & Wetland \\
\hline 01BL-24B & $\begin{array}{l}\text { Reddish, blocky silty clay and sand } \\
\text { layers, rare shells }\end{array}$ & $4.2-6.1 \mathrm{~m}$ & & None & $\begin{array}{l}\text { Terrestrial } \\
\text { gastropods, } \\
\text { seeds }\end{array}$ & $\begin{array}{l}\text { Environmental setting unknown, wet ground } \\
\text { possible }\end{array}$ \\
\hline 01BL-24A & $\begin{array}{l}\text { Thinly bedded well sorted silt and } \\
\text { fine sand between beach gravel } \\
\text { beds, rare shells }\end{array}$ & $6.6-7.3 \mathrm{~m}$ & & $\begin{array}{l}\text { Candona rawsoni } \\
\text { Cytherissa lacustris } \\
\text { Candona caudata } \\
\text { Physocypria globula } \\
\text { Limnocythere paraornata }\end{array}$ & $\begin{array}{l}\text { Aquatic } \\
\text { gastropods, } \\
\text { bivalves }\end{array}$ & $\begin{array}{l}\text { Lacustrine, but likely with a nearby stream or } \\
\text { through-flowing wetland setting, such as } \\
\text { might exist in a marginal lacustrine site like } \\
\text { modern-day Mud Lake }\end{array}$ \\
\hline
\end{tabular}


Table 3. Ostracodes in sediment samples from outcrops in the Bear Lake drainage basin, 1998-2001-Continued.

\begin{tabular}{|c|c|c|c|c|c|c|}
\hline $\begin{array}{l}\text { Site and } \\
\text { sample } \\
\text { number }\end{array}$ & $\begin{array}{l}\text { Description of unit } \\
\text { (see table 1) }\end{array}$ & $\begin{array}{l}\text { Depth below } \\
\text { top of } \\
\text { section (cm } \\
\text { unless } \\
\text { noted) }\end{array}$ & $\begin{array}{l}\text { Age } \\
\text { (if dated; } \\
\text { see table } \\
\text { 2) }\end{array}$ & Ostracode taxa & Other taxa & Comments \\
\hline 01BL-30A & $\begin{array}{l}\text { Pale olive-green sandy silt, well- } \\
\text { bedded, noncalcareous }\end{array}$ & $285-300$ & & None & None & $\begin{array}{l}\text { Sample contains volcanic ash, most grains are clear } \\
\text { to frosty, rare grains are black }\end{array}$ \\
\hline 01BL-35B & $\begin{array}{l}\text { Marl; overlies well-sorted, fine- } \\
\text { medium crossbedded sand }\end{array}$ & $150-190$ & $13,675 \pm 50$ & $\begin{array}{l}\text { Heterocypris } \mathrm{sp} . \\
\text { Candona } \mathrm{sp} . \\
\text { Limnocythere paraoranata }\end{array}$ & Aquatic snails & Spring seep complex \\
\hline 01BL-39C & $\begin{array}{l}\text { Well-sorted, fine reddish sand fining } \\
\text { upward to sandy silt, about } 8 \mathrm{~m} \text { thick; } \\
\text { underlain by Bear River terrace gravel }\end{array}$ & $\sim 6 \mathrm{~m}$ & & None & $\begin{array}{l}\text { Terrestrial } \\
\text { gastropods, } \\
\text { insects }\end{array}$ & Environment unknown, wet ground possible \\
\hline $\begin{array}{l}\text { 01BL-42 } \\
\text { old sample } \\
\text { 99BL58B }\end{array}$ & $\begin{array}{l}\text { Well- sorted, interbedded, reddish } \\
\text { sand, silt, and mud layers, locally } \\
\text { reduced greenish color }\end{array}$ & $22-22.7 \mathrm{~m}$ & $\begin{array}{l}<760 \mathrm{ka} \\
\text { (Bishop ash } \\
\text { bed) }\end{array}$ & $\begin{array}{l}\text { Limnocythere sp.? } \\
\text { Limnocythere staplini? } \\
\text { Candonids, ground-water } \\
\text { taxa }\end{array}$ & & $\begin{array}{l}\text { Environment unknown, could be related to a fluvial } \\
\text { or alluvial aquifer }\end{array}$ \\
\hline $\begin{array}{l}\text { 01BL-42 } \\
\text { old sample } \\
\text { 99BL58A }\end{array}$ & $\begin{array}{l}\text { Well-sorted, interbedded, reddish sand, } \\
\text { silt, and mud layers, locally reduced } \\
\text { greenish color }\end{array}$ & $22-22.7 \mathrm{~m}$ & $\begin{array}{l}<760 \mathrm{ka} \\
\text { (Bishop ash } \\
\text { bed) }\end{array}$ & Candona rawsoni & & $\begin{array}{l}\text { Material is composed of only broken shells of } \\
\text { juveniles; they looked reworked }\end{array}$ \\
\hline 01BL-42C & $\begin{array}{l}\text { Marl, 3-cm thick in sequence of } \\
\text { reddish, thinly bedded fine silty sand }\end{array}$ & $\sim 24 \mathrm{~m}$ & $\begin{array}{l}<760 \mathrm{ka} \\
\text { (Bishop ash } \\
\text { bed) }\end{array}$ & $\begin{array}{l}\text { Limnocythere ceriotuberosa } \\
\text { Candona sp. cf. C. rawsoni }\end{array}$ & & $\begin{array}{l}\text { Ostracodes are reworked. Limnocythere } \\
\text { ceriotuberosa was a common ostracode in most of } \\
\text { the deep lakes that resided in the greater Great Salt } \\
\text { Lake Basin }\end{array}$ \\
\hline DK93-23E & $\begin{array}{l}\text { Peat and organic-rich silt with shell- } \\
\text { hash layers }\end{array}$ & $0-100$ & & $\begin{array}{l}\text { Physocypira } \\
\text { Limnocythere } \sim \text { Itasca } \\
\text { Cyclocypris } \\
\text { Candona distincta } \\
\text { Candona renoensis } \\
\text { Candona acuminata } \\
\text { Potamocypris } \\
\text { Candona sp. (caudatid?) }\end{array}$ & & \\
\hline DK93-23D & $\begin{array}{l}\text { Dark gray, fine and medium sand; } \\
\text { layers of snails }\end{array}$ & $100-170$ & $6750 \pm 60$ & $\begin{array}{l}\text { Physocypira } \\
\text { Limnocythere } \sim \text { Itasca } \\
\text { Cyclocypris } \\
\text { Cypridopsis vidua } \\
\text { Potamocypris } \\
\text { Candona renoensis } \\
\text { Candona } \sim \text { candida } \\
\text { Candona } \mathrm{n} \text {. sp. } 1 \text { and } 2\end{array}$ & $\begin{array}{l}\text { Carinifex, } \\
\text { Lymnaea } \text { snails }\end{array}$ & $\begin{array}{l}\text { Candona n. sp. } 1 \text { and } 2 \text { are the two most common } \\
\text { Bear Lake endemics. Also, possibly modified } \\
\text { versions of Candona } \text { n. sp. } 3 \text { and } 4 \text { from Bear } \\
\text { Lake }\end{array}$ \\
\hline DK93-23C & $\begin{array}{l}\text { Organic-rich silt and peat with silty } \\
\text { sand layers; snail shells and shell hash }\end{array}$ & $170-190$ & & $\begin{array}{l}\text { No adult ostracodes, few } \\
\text { juveniles }\end{array}$ & & \\
\hline
\end{tabular}


Table 3. Ostracodes in sediment samples from outcrops in the Bear Lake drainage basin, 1998-2001-Continued.

\begin{tabular}{|c|c|c|c|c|c|c|}
\hline $\begin{array}{l}\text { Site and } \\
\text { sample } \\
\text { number }\end{array}$ & $\begin{array}{l}\text { Description of unit } \\
\text { (see table 1) }\end{array}$ & $\begin{array}{l}\text { Depth below } \\
\text { top of } \\
\text { section (cm } \\
\text { unless } \\
\text { noted) }\end{array}$ & $\begin{array}{l}\text { Age } \\
\text { (if dated; } \\
\text { see table } \\
\text { 2) }\end{array}$ & Ostracode taxa & Other taxa & Comments \\
\hline DK93-23B & $\begin{array}{l}\text { Laminated marl, rhythmically bedded } \\
\text { gray silt and white silt-clay; abundant } \\
\text { snails }\end{array}$ & $190-270$ & & $\begin{array}{l}\text { Cypridopsis vidua } \\
\text { Physocypria } \\
\text { Limnocythere } \sim \text { Itasca } \\
\text { Candona compressa } \\
\text { Candona } \text { n. sp. } 2\end{array}$ & & $\begin{array}{l}\text { Candona } \text { n. sp. } 2 \text { is the most common endemic in } \\
\text { Bear Lake. Few valves }\end{array}$ \\
\hline DK93-23A & Sedge peat & $270-280$ & & $\begin{array}{l}\text { Cypridopsis vidua } \\
\text { Physocypria } \\
\text { Candona } \sim \text { candida } \\
\text { Limnocythere } \sim \text { itasca }\end{array}$ & & Very few valves \\
\hline DK96-06 & $\begin{array}{l}\text { Brown mud; top of tube driven below } \\
\sim 150 \mathrm{~cm} \text { in section at Fish Ladder }\end{array}$ & $0-10$ in tube & $\geq 8550$ & $\begin{array}{l}\text { Physocypria } \\
\text { Cypridopsis vidua } \\
\text { Cyclocypris ampla } \\
\text { Candona sp.? (caudatid) } \\
\text { Limnocythere itasca or } \\
\text { itascoid } \\
\text { Limnocythere sp.? }\end{array}$ & $\begin{array}{l}\text { Valvata, Gyraulis } \\
\text { snails }\end{array}$ & \\
\hline DK96-06 & Deformed, pasty gray marl & 11 in tube & $\geq 8550$ & $\begin{array}{l}\text { Physocypria } \\
\text { Candona sp.? (caudatid) } \\
\text { Limnocythere Itasca } \\
\text { Candona compressa } \\
\text { Candona acuminata } \\
\text { Cypridopsis vidua }\end{array}$ & $\begin{array}{l}\text { Lymnaea, some } \\
\text { Valvata snails }\end{array}$ & \\
\hline DK96-06 & Dark gray mud & 21 in tube & $\geq 8550$ & $\begin{array}{l}\text { Candona sp.? (caudatid) } \\
\text { Physocypria } \\
\text { Cypridopsis vidua } \\
\text { Cyclocypris } \\
\text { Limnocythere itasca }\end{array}$ & $\begin{array}{l}\text { Valvata, Gyraulus } \\
\text { snails; Pisidium } \\
\text { or Sphaerium } \\
\text { clams }\end{array}$ & \\
\hline DK96-06 & Laminated, gray to light tan mud & 26 in tube & $\geq 8550$ & $\begin{array}{l}\text { Limnocythere } \sim \text { itasca } \\
\text { Physocypria } \\
\text { Candona sp.? (caudatid) } \\
\text { Cypridopsis vidua }\end{array}$ & $\begin{array}{l}\text { Gyraulus, Valvata } \\
\text { snails; Pisidium } \\
\text { or Sphaerium } \\
\text { clams; } \\
\text { charophytes }\end{array}$ & \\
\hline DK96-06 & $\begin{array}{l}\text { Black, gritty, stiff mud; base of tube } \\
\text { driven below } \sim 150 \mathrm{~cm} \text { in section at } \\
\text { Fish Ladder }\end{array}$ & 31 in tube & $\geq 8550$ & None & None & Sieve residue is charcoal and burned seeds \\
\hline $\begin{array}{r}\text { DK99-28 } \\
\text { (same as }\end{array}$ & Gray, massive marl & $50-200$ & $>45,000$ & None & Stagnicola & \\
\hline
\end{tabular}


Table 3. Ostracodes in sediment samples from outcrops in the Bear Lake drainage basin, 1998-2001-Continued.

\begin{tabular}{|c|c|c|c|c|c|c|}
\hline $\begin{array}{l}\text { Site and } \\
\text { sample } \\
\text { number }\end{array}$ & $\begin{array}{l}\text { Description of unit } \\
\text { (see table 1) }\end{array}$ & $\begin{array}{l}\text { Depth below } \\
\text { top of } \\
\text { section (cm } \\
\text { unless } \\
\text { noted) }\end{array}$ & $\begin{array}{l}\text { Age } \\
\text { (if dated; } \\
\text { see table } \\
\text { 2) }\end{array}$ & Ostracode taxa & Other taxa & Comments \\
\hline $\begin{array}{l}\text { DK99-32A } \\
\text { (same as } \\
\text { 99BL-53) }\end{array}$ & $\begin{array}{l}\text { Well-sorted well-bedded fine-medium } \\
\text { sand and silt }\end{array}$ & $\sim 16 \mathrm{~m}$ & & None & None & \\
\hline BL00-13 & Multiple marl beds & $115-260$ & & Candona sp.? & Sphaerium & \\
\hline BL00-17 & $\begin{array}{l}\text { Yellow, massive, slightly weathered } \\
\text { marl with Stagnicola shells }\end{array}$ & $90-355$ & & None & $\begin{array}{l}\text { Stagnicola, } \\
\text { Gyraulus, } \\
\text { Discus, and } \\
\text { Valvata }\end{array}$ & \\
\hline BL00-18 & Gray, massive marl & $240-250$ & & $\begin{array}{l}\text { Strandesia meadensis } \\
\text { Cavernocypris } \\
\text { Cypridopsis }\end{array}$ & None & \\
\hline BL00-19 & $\begin{array}{l}\text { Green, massive marl with Stagnicola } \\
\text { shells }\end{array}$ & $175-250$ & & None & Stagnicola & \\
\hline
\end{tabular}


Figure 1. Map of Bear Lake Valley showing site locations and generalized traces of Quaternary faults and modern and abandoned stream courses. Site numbers beginning with year (for example, 99BL-35) were described and sampled by Reheis; prefixes for some sites were omitted for ease of drafting, but all the omitted prefixes are for sites described in 1998 (for example, site 98BL-47 is abbreviated to 47). Site numbers beginning with $\mathrm{BL}$ - were described and sampled by Laabs; those beginning with $\mathrm{DK}$ - were described and sampled by Kaufman. A, airport; B, Bloomington; D, Dingle; GC, Garden City; M, Montpelier; P, Paris; SC, St. Charles.

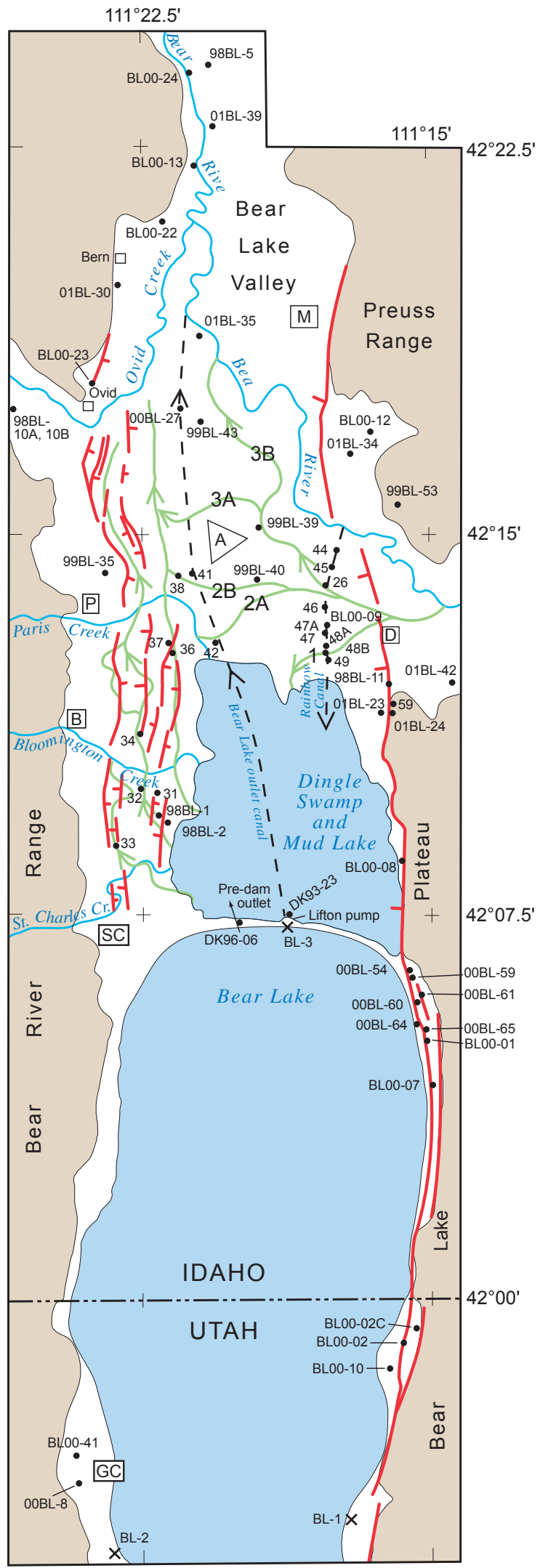

\section{EXPLANATION}

\begin{tabular}{|c|c|}
\hline & Bedrock \\
\hline C & Modern drainage \\
\hline & Lake or marsh \\
\hline & Abandoned channel \\
\hline & Fault and sense of displacement \\
\hline $98 \stackrel{\bullet}{\mathrm{BL}-8}$ & $\begin{array}{l}\text { Stratigraphic section (see table 1); } \\
\text { number alone is abbreviated from } \\
99 B L-\text { for ease of drafting }\end{array}$ \\
\hline$\underset{B L-1}{X}$ & Dust trap locality \\
\hline $\mathrm{M}$ & Town or airport \\
\hline
\end{tabular}

Index to $7.5^{\prime}$ quadrangle maps

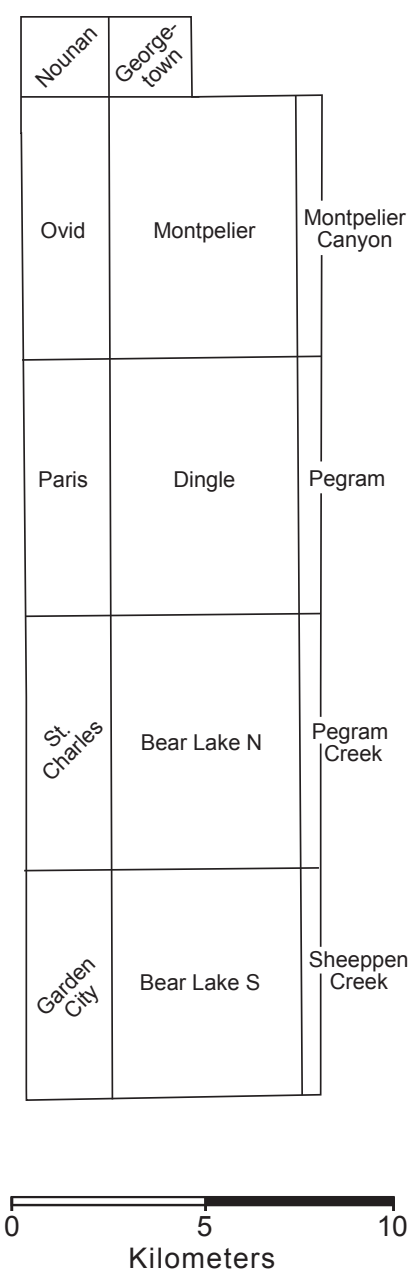




\title{
EXPLANATION FOR BEAR LAKE SECTIONS
}

\author{
Site number (type of exposure) \\ Surface altitude (in meters and feet; \\ annotated if measured using GPS) \\ Mud or clay \\ Marl \\ Interbedded mud and sand \\ Silt and sand, well sorted \\ and bedded \\ Fine sand and silt, well sorted, \\ massive (loess) \\ Sand and gravel, well \\ rounded clasts (beach \\ and nearshore deposits) \\ Crossbedded gravel and \\ sand, rounded clasts \\ (fluvial deposits) \\ Crossbedded sand and fine \\ pebble gravel (fluvial deposits)

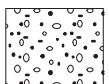 \\ Clay, silt, sand, and minor pebbles, \\ moderately sorted (fluvial and \\ overbank deposits) \\ Peat and organic-rich \\ deposits \\ Clay, silt, sand, and gravel, \\ poorly sorted and bedded \\ (alluvial fan deposits) \\ Steep dips (foreset beds) \\ 亡ー Calcareous cement, locally tufa \\ $\sum \zeta \zeta$ Buried soil \\ Erosional contact \\ Btk Soil horizon \\ $\leftarrow$ A $2445{ }^{14} \mathrm{C}$ yr B.P. Sample letter or depth in centimeters \\ and ${ }^{14} \mathrm{C}$ age (if dated) \\ $01 B L 42$ \\ Tephra layer and \\ sample number
}

Figure 2. Stratigraphic sections measured by Reheis from outcrops and auger holes in Bear Lake valley. Site locations are in figure 1 and table 1. Sections are shown in rough order from north to south on the following four pages. Column to right of lithology gives descriptive information such as color, sample data, and soil horizons. On some sections, leaders group depositional layers into different types of deposits (e.g., loess) as interpreted from sedimentary characteristics. 
Figure 2. Continued.
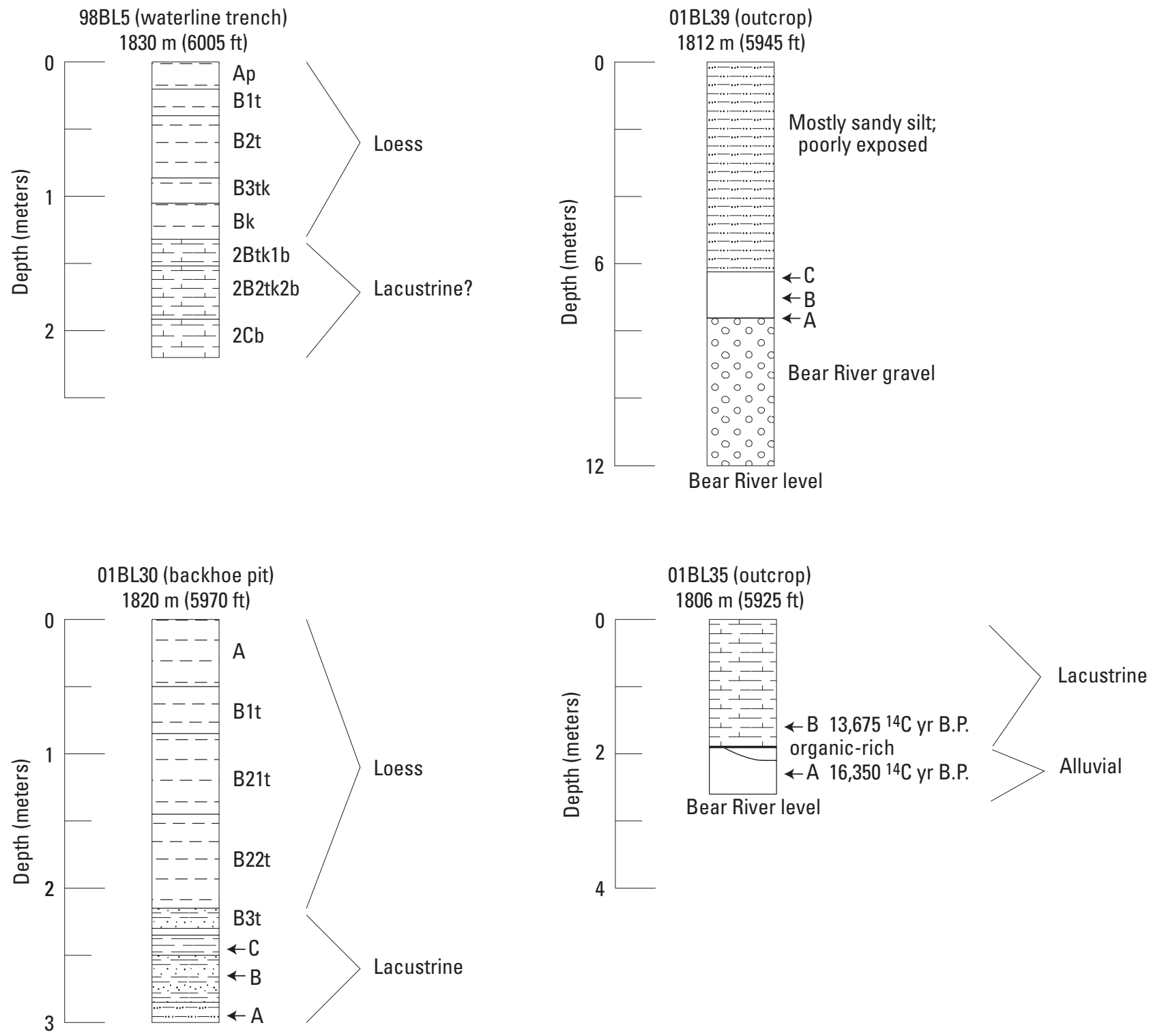

98BL10A (auger in sinkhole) $1813 \mathrm{~m}$ (5949 ft)

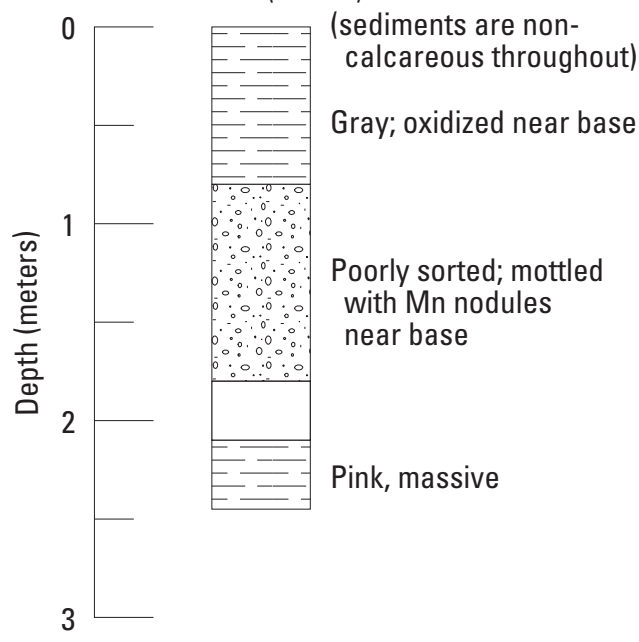

98BL10B (auger on sinkhole rim) $1815 \mathrm{~m}(5955 \mathrm{ft})$

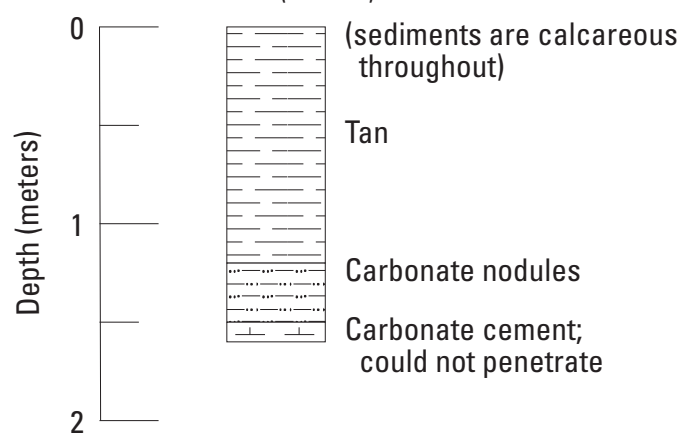


Figure 2. Continued.
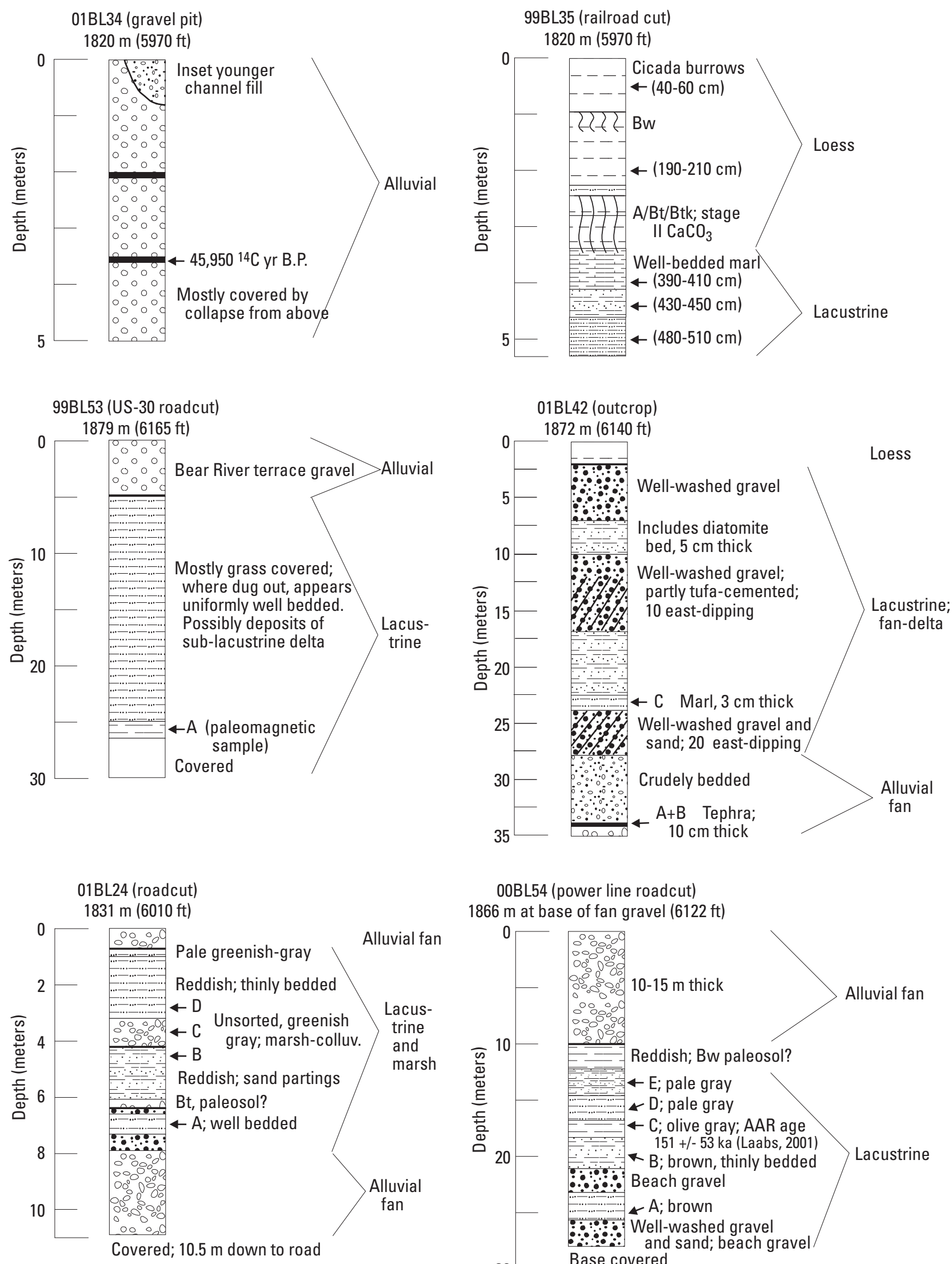

00BL54 (power line roadcut)

$1866 \mathrm{~m}$ at base of fan gravel (6122 ft)

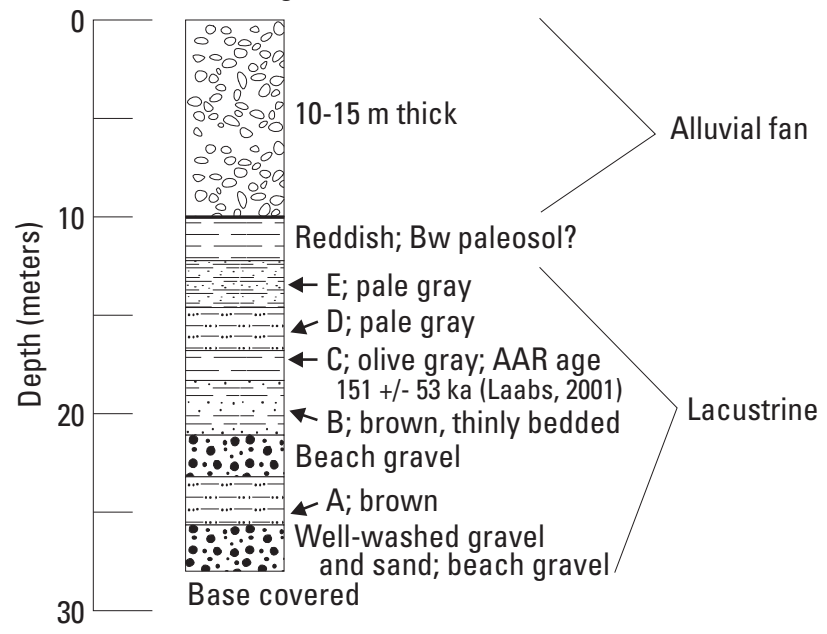


Figure 2. Continued.
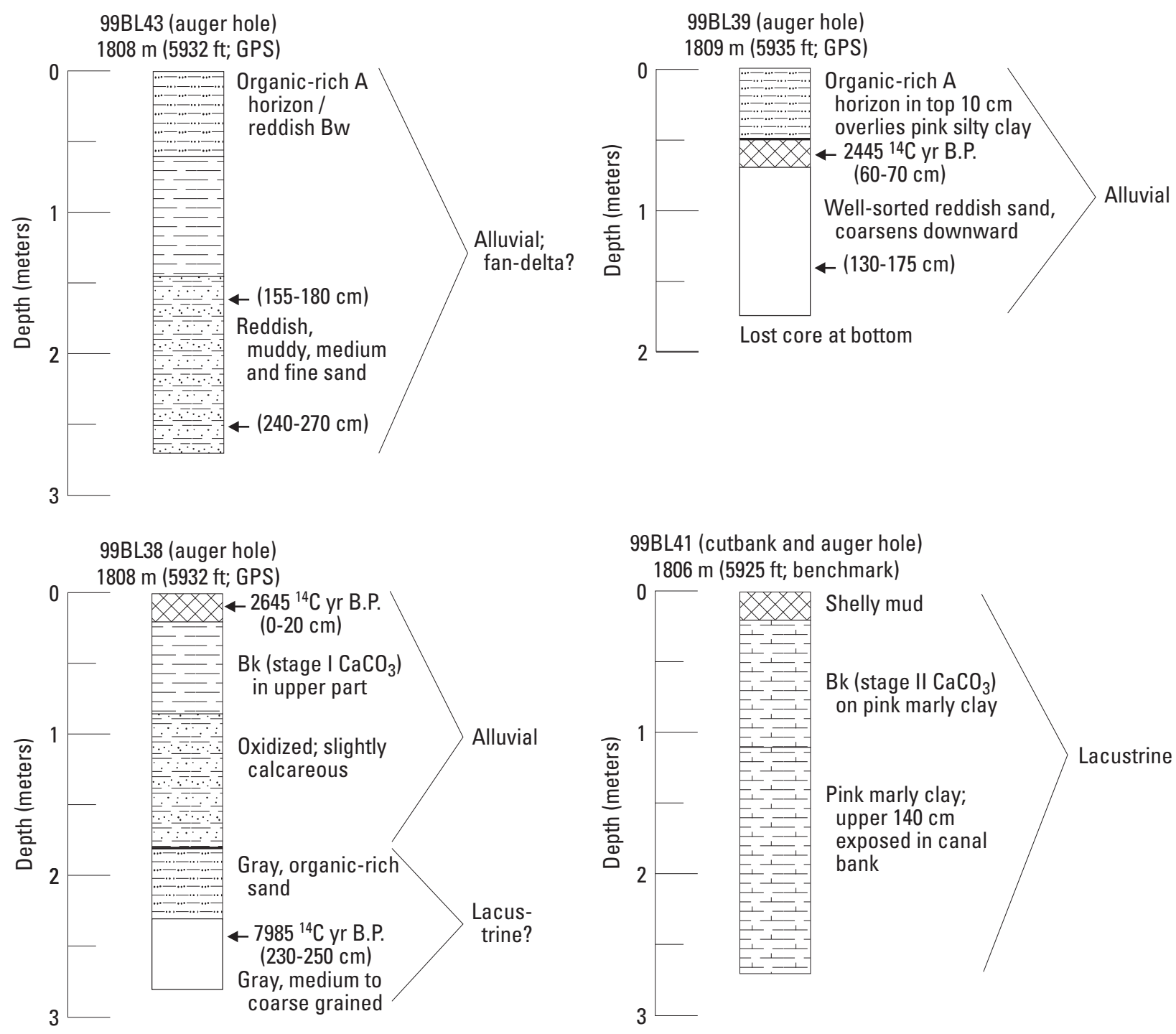

99BL41 (cutbank and auger hole) $1806 \mathrm{~m}$ (5925 ft; benchmark)
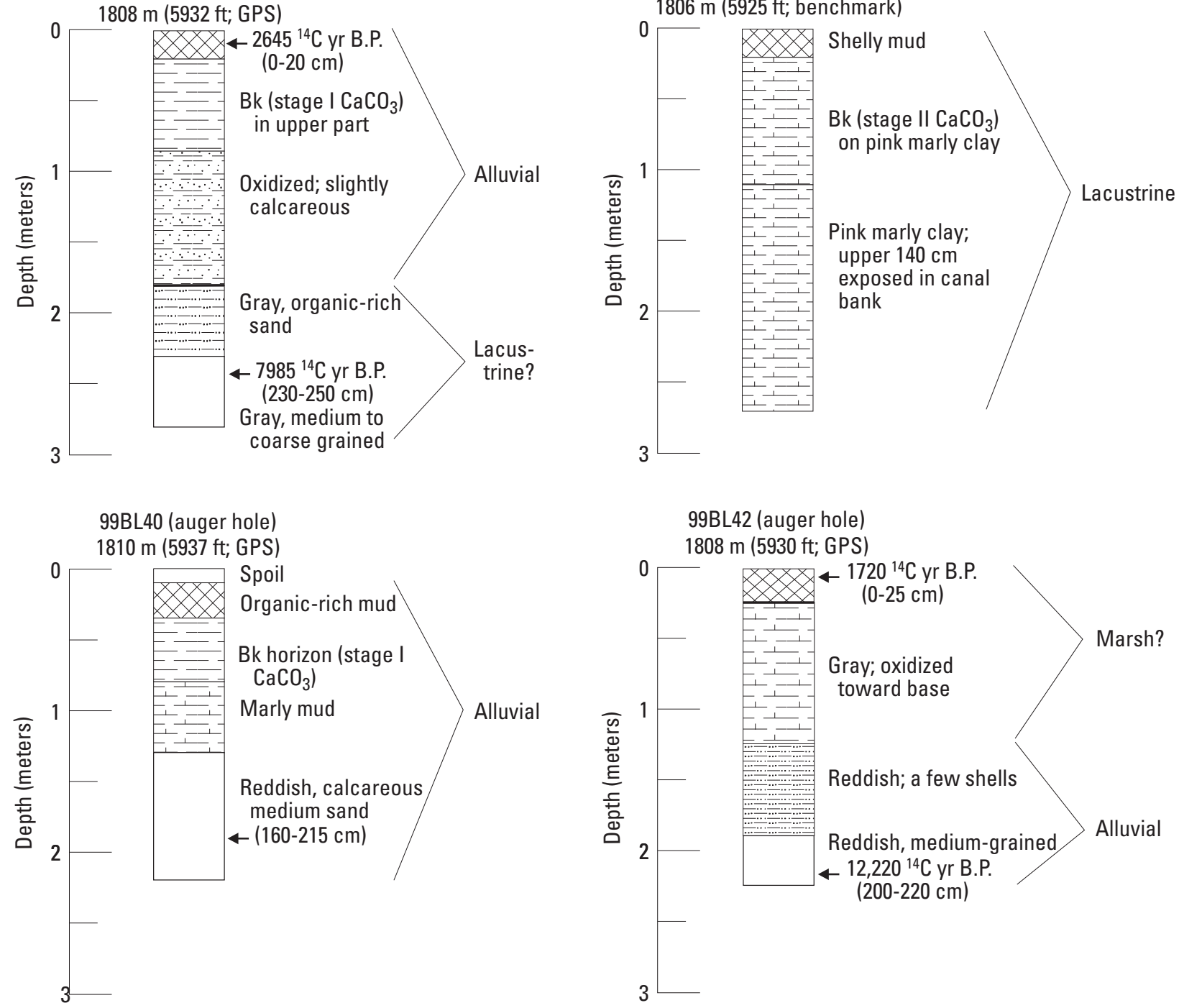
Figure 2. Continued.
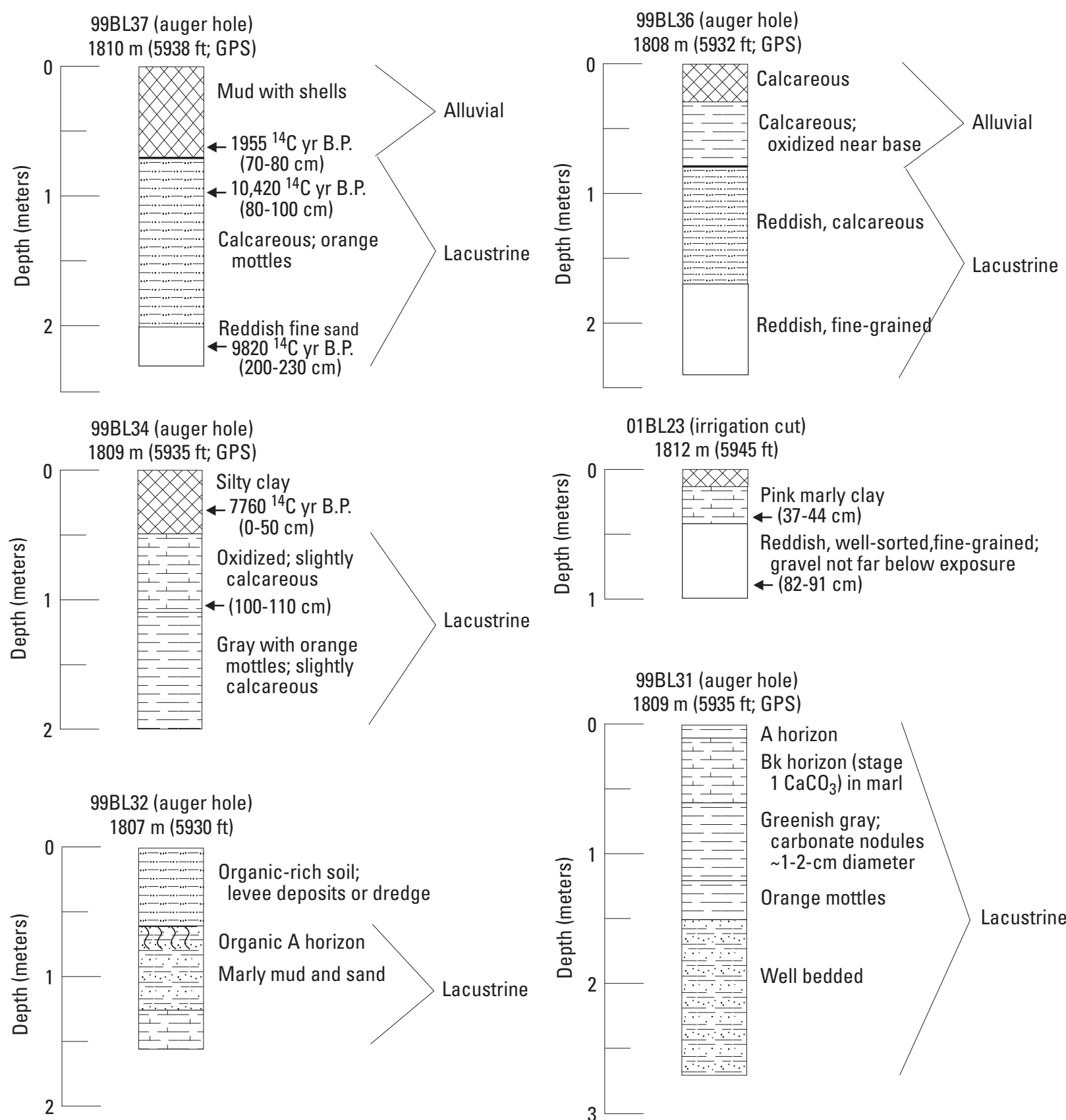

Organic-rich soil; levee deposits or dredge
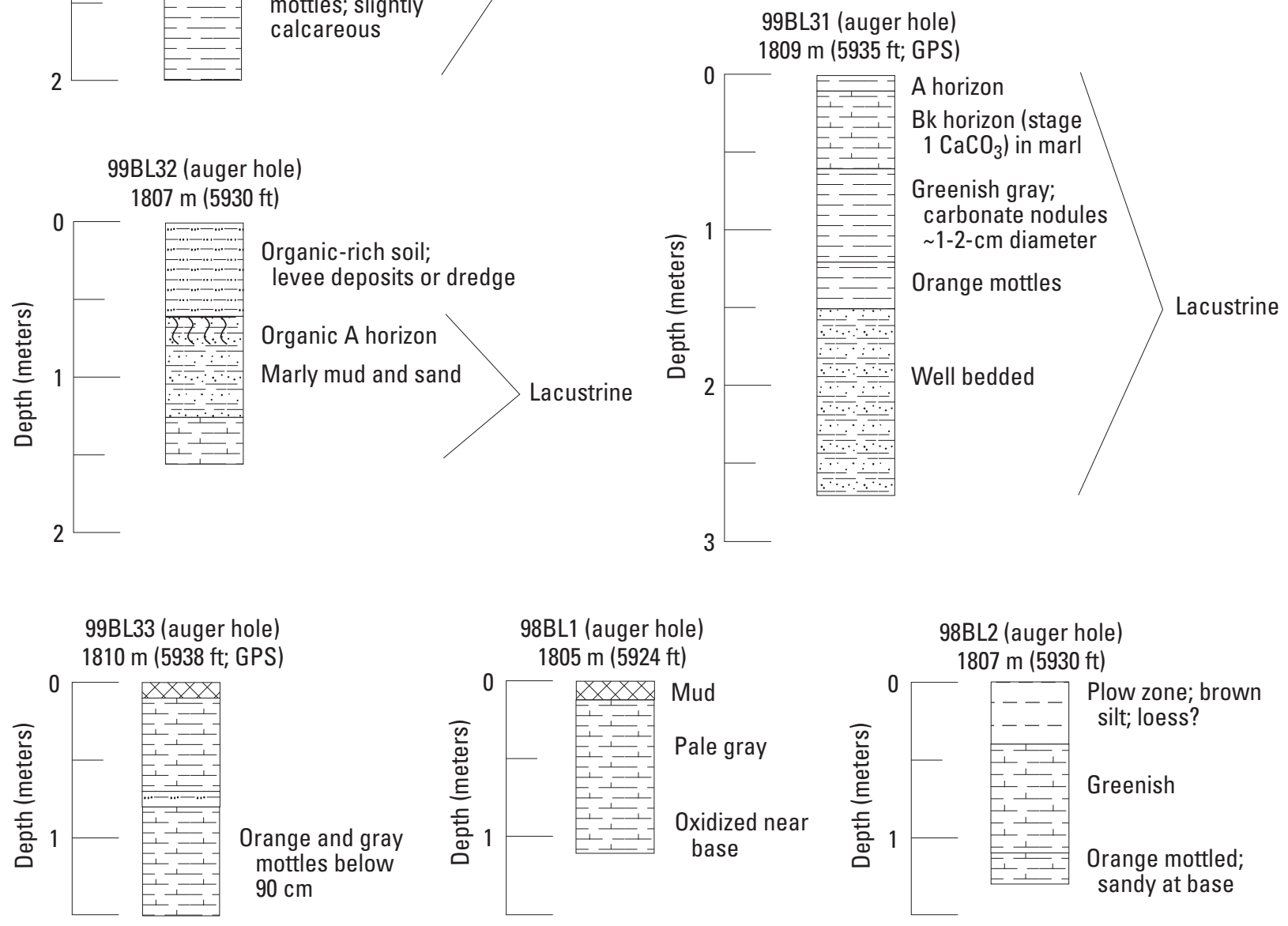


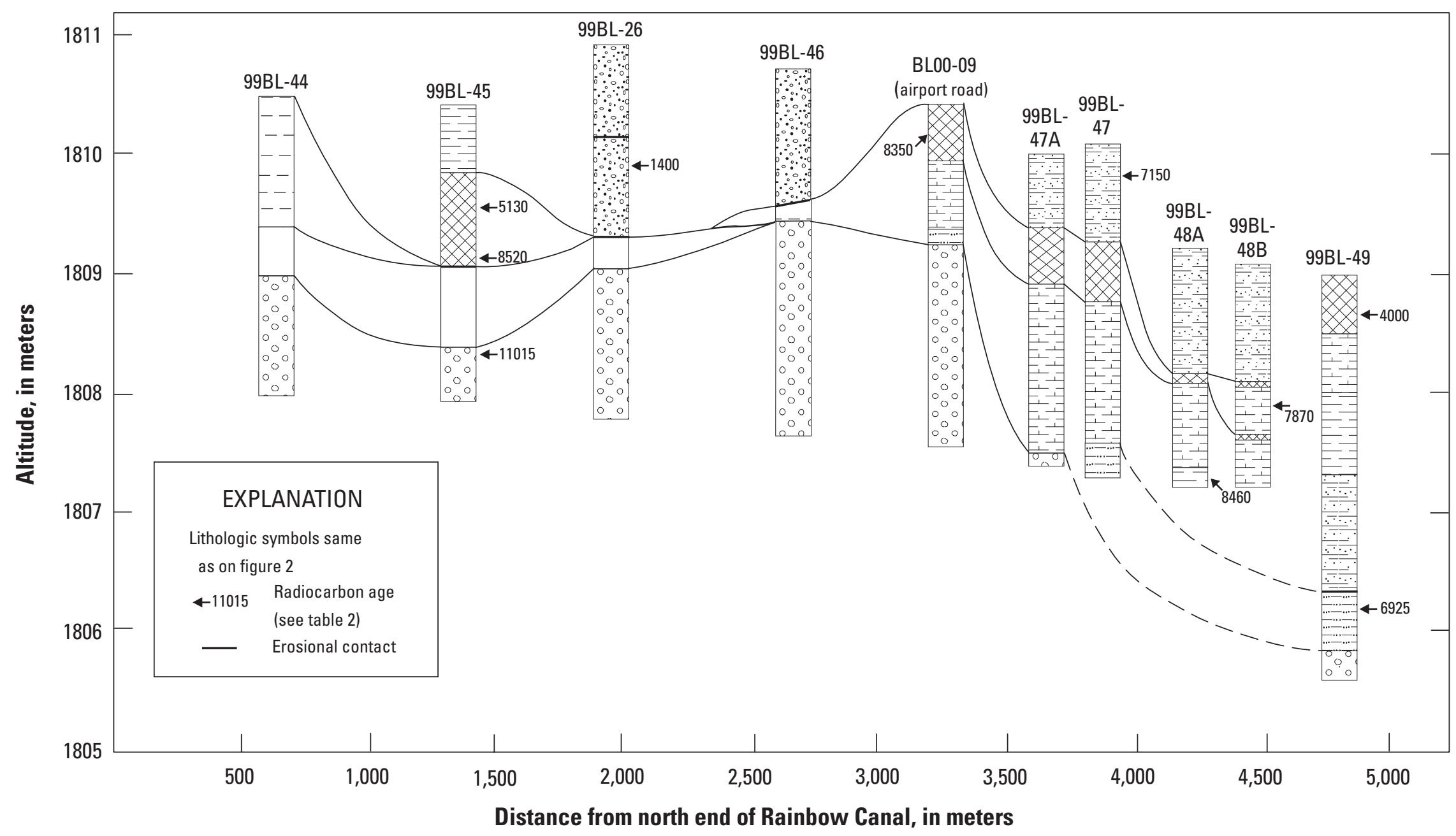

Figure 3. Stratigraphic sections of exposures along the Rainbow Canal (see table 1). Most sections are outcrops and correlation lines are physically traced, except that BL99-49 is an auger hole within a channel fill cut into older sediment and correlations are uncertain. Surface altitudes were measured using a Trimble GPS and differentially corrected in the office. The base of the outcrop sections is the water level, thus the section bases essentially reflect the water gradient in the canal over a two-day period when flow rate in the canal remained relatively constant. Measured sections were then plotted and their altitudes slightly adjusted to yield a smoothly sloping water level at the base of the outcrop sections. 